\title{
Preliminary design, flow field, and thermal performance analysis of a helium turboexpander: a numerical approach
}

\author{
Manoj Kumar $^{1}$ - Debashis Panda ${ }^{1} \cdot$ Amitesh $_{\text {kumar }^{2} \cdot \text { Ranjit K. Sahoo }^{1} \cdot \text { Suraj K. Behera }}{ }^{1}$
}

Received: 2 August 2019 / Accepted: 14 October 2019 / Published online: 24 October 2019

(c) Springer Nature Switzerland AG 2019

\begin{abstract}
Most studies on cryogenic turboexpanders are focused on parametric studies and mean-line design to increase the performance of cryogenics liquefaction cycle without much attention to the splitter blades which are crucial for the stability of the flow field. This study focuses on a novel mean-line design methodology to develop the radial turbine and nozzle (hereafter renowned as turboexpander) to investigate the performance characteristics. Firstly, Sobol sensitivity analysis is performed to identify the effect of major non-dimensional design variables on isentropic efficiency of the turbine. Secondly, the non-dimensional design variables are optimized using artificial intelligence techniques. Thirdly, three turboexpander models with and without splitter blades are designed within the optimized range of non-dimensional variables. After that, the three-dimensional numerical analysis is carried out to visualize the effect of splitter blades on flow field and thermal characteristics of the turboexpander. It is noticed that the passage vortices and flow separation are minimized using the splitter blades. The numerical results are further validated with available data in the literature. A detailed comparative analysis of Mach number, pressure, temperature, velocity, static enthalpy, static entropy, etc., is carried out at different operating conditions. The results reveal that the use of splitter blades has a tremendous effect on the performance and flow field characteristics of the radial turbine. The proposed methodology specifies the insights for an optimum turbine design methodology of a cryogenic turboexpander, Sobol sensitivity analysis, prediction capability of artificial intelligence methods, numerical techniques to simulate the assimilating performance of turboexpander, as it is the most crucial and expensive component of turboexpander-based cryogenic system.
\end{abstract}

Keywords Helium turboexpander · Splitter blade · Sobol method · Flow pattern · Thermal characteristics · Artificial intelligence

\section{List of symbols}

Variables

$b \quad$ Blade height $(\mathrm{m})$

C Absolute velocity $(\mathrm{m} / \mathrm{s})$

D Turbine diameter ratio

d diameter $(\mathrm{m})$

$D_{i}(Y) \quad$ First-order variance

$D_{i j}(Y) \quad$ Second-order variance

$d_{s} \quad$ Specific diameter

$h \quad$ Enthalpy $(\mathrm{kJ} / \mathrm{kg})$

$k_{0} \quad$ Thermal conductivity of fluid $(\mathrm{W} / \mathrm{m} \mathrm{K})$
$L_{\mathrm{t}} \quad$ Total losses

$M \quad$ Mach number

$\dot{m} \quad$ Mass flow rate $(\mathrm{kg} / \mathrm{s})$

$n_{\mathrm{s}} \quad$ Specific speed

$P \quad$ Power (kW)

$p \quad$ Pressure $(\mathrm{Pa})$

$r_{\mathrm{p}} \quad$ Pressure ratio

$S_{i} \quad$ First-order sensitivity index

$T \quad$ Temperature (K)

$U \quad$ Blade speed $(\mathrm{m} / \mathrm{s})$

$\operatorname{Var}(Y)$ Total variance of the output

Manoj Kumar, manojbeg526@gmail.com | ${ }^{1}$ National Institute of Technology Rourkela, Rourkela, Odisha 769008, India. ${ }^{2}$ Indian Institute of Technology Varanasi, Varanasi, U.P 221005, India. 
$v_{\mathrm{s}} \quad$ Velocity ratio

Z Number of blades

\section{Greek symbols}

$\alpha \quad$ Absolute flow angle $\left({ }^{\circ}\right)$

$\beta \quad$ Relative flow angle $\left({ }^{\circ}\right)$

$\varepsilon_{\mathrm{x}} \quad$ Axial clearance (m)

$\varepsilon_{\mathrm{r}} \quad$ Radial clearance (m)

$\lambda \quad$ Ratio of rotor outlet to inlet

$\mu \quad$ Coefficient of viscosity (Pa s)

$\Omega \quad$ Degree of reaction

$\phi \quad$ Flow coefficient

$\psi \quad$ Stage head coefficient

$\chi \quad$ Absolute meridional velocity

$\eta_{\text {is }} \quad$ Isentropic efficiency

$\omega \quad$ Rotational speed (rpm)

\section{Subscripts}

2 Turbine inlet

ex Turbine exit

in Nozzle inlet

$s \quad$ Isentropic

\section{Acronyms}

ANFIS Adaptive neuro-fuzzy inference system

ANN Artificial neural network

MF Membership function

ORC Organic Rankine cycle

PS Pressure surface

RMSE Root mean squared error

SS Suction surface

SST Shear stress transport

TF Transfer function

TKE Turbulence kinetic energy

\section{Introduction}

The advancement of cryogenic science needs more attention to achieve the liquid helium temperature region. The efficient helium refrigeration and liquefaction systems are necessary because of its applications in high energy physics, fusion reactors, particle accelerators, space industries, power engineering, biomedical and chemical industries. The requirements of liquid helium can be fulfilled by developing an efficient turboexpander system in which turbine and nozzle are the major components. Generally, these micro-turbines are radial inflow type due to low molecular weight and density of the helium fluid [1]. Since these turbines are operated at high rotational speeds, the size of the turbine tends to be small which leads to mechanical design challenges. However, recent development in small scale radial turbine redraws the interest to develop an efficient turbine by using the optimum range of design parameters. Additionally, the advantages of compact size, operation stability, and a wide range of applications, radial turbines can be used in gas liquefaction system, ORC cycles, air separation plants, etc. [2-4]. The primary purpose of reverse Brayton and Claude cycle-based turboexpander is to produce the cryogenic temperature. Therefore, its performance determines the efficiency of the system. An appropriate design of the turbine blade profile defines its efficiency and operational stability.

Ghosh et al. $[5,6]$ conducted an experimental and analytical study for testing a high-speed cryogenic turboexpander. Hongli et al. [7] performed the experimental analysis of a reversed Brayton cycle-based high-speed cryogenic turboexpander. Sam et al. [8] studied the effect of turbine rotation on the fluid flow characteristics of highspeed helium turbine. Zhang et al. [9] developed a turboexpander for power recovery in refrigerating systems. Davis and Land [10] propose the power recovery by the compressor from the turboexpander system.

A detailed mean-line methodology and parametric analysis are conducted to obtain the maximum efficiency of a radial inflow turbine used in an organic Rankine cycle [11-14]. Some authors suggest the various well-established loss model correlations, and some modifications are summarized for accurate and robust design of small scale radial turbine, but none of them reported to use these methodologies for cryogenic turbine $[15,16]$. The different loss models must be considered in the design process of a turboexpander, in particular for cryogenic applications which may increase the efficiency of the turbine in particular, and the same for the system in general. Recently, Mounier et al. [17] design small turboexpander for an ORC system by adding supplements (design variable optimization) in the thermodynamic and preliminary design process. Generally, the turbine design methodology depends on the initial guess of isentropic efficiency. It needs to be modified with a detailed design process considering various losses, operating conditions, and real gas properties of the working fluid. Several works report the usage of ANN models for obtaining the best blade profile of gas turbines. Some of them apply this methodology in turbomachinery as well as to investigate the performance of gas turbines, and compressors [18-20].

A detailed literature review suggests that there are a plethora of works available for the design methodology of the radial turbine, but none of them apply Sobol sensitivity test to identify major non-dimensional variables and predicting their optimal range using artificial intelligence techniques. Additionally, the effect of splitter blade on the performance of the turboexpander is also analyzed. It is useful for flow stability at the outlet of the turbine which is essential for reducing the entropy generation [21, 22]. This paper attempts to overwhelm this literature gap. 
In this paper, the mean-line design methodology of the cryogenic radial turbine and the nozzle is explained. The aerodynamic and profile design methodology of a radial inflow turbine and nozzle is focused. The important nondimensional design variable datasets are used to develop an artificial neural network (ANN) and adaptive neurofuzzy inference system (ANFIS) network which predicts their optimal ranges, in which the performance of the turbine is maximum. Based on the predicted ranges, three turboexpander models (high, medium, and low pressure) are developed. After that, the numerical simulations are carried out to visualize the fluid flow and thermal characteristics using commercial CFD platform ANSYS`CFX. The Peng-Robinson real gas formulation is opted to achieve the accurate thermal performance and flow features of the working fluid. Then, the numerical results are validated with available data in the open literature. The fluid flow such as velocity, Mach number, pressure variation, Reynolds number, etc., and thermal parameters such as static enthalpy, static entropy, temperature variation, Prandtl number, etc., are obtained at various spans and along the streamwise locations. Furthermore, the analysis is extended to reveal the effect of the splitter blades on the performance of the turboexpander. The results are useful for mean-line design, performance prediction, and development of a modified Claude cycle-based cryogenic turboexpander for the liquefaction of helium.

The paper is organized as follows: Sect. 2 illustrates the design methodology, Sobol sensitivity analysis. Section 3 discusses the ANN and ANFIS model development and predicted results. Section 4 illustrates the numerical techniques, boundary condition, and turbulence model schemes. Section 5 illustrates the results and discussions. Section 6 concludes the results.

\section{Preliminary design procedure}

\subsection{An approach to design a turboexpander-based helium liquefaction system}

Figure 1 depicts the design methodology of a helium liquefaction system which is based on a modified Claude cycle. It contains a helium compressor, cryogenic valves, helium turboexpanders, heat exchangers, reservoir, etc. There are three turboexpanders (high, medium, and low pressure) used in this system. The helium is compressed to high pressure (17 bar), then precooled in the HEX 1 which is equipped with liquid nitrogen. In this way, the highpressure and low-temperature helium passes through the first (high-pressure) turboexpander $(T 1)$. To overcome the turbulence and vortices at the outlet of the first stage expansion, HEX 2 is placed in which the subcooling of the fluid takes place. After that, the fluid ( 8 bar, $60 \mathrm{~K}$ ) passes through the medium pressure turboexpander ( $T 2)$ and low-pressure turboexpander ( $4.5 \mathrm{bar}$ and $48 \mathrm{~K}$ ). The Joule-Thomson valve is placed after the HEX 4, and the process is repeated.

\subsection{Preliminary design of radial inflow turbine}

The preliminary design of the turbine blade geometry begins with the input datasets (thermodynamic parameters) and some non-dimensional parameters within a specified range. There are excessive empirical correlations enlisted; the mean-line design procedure is initiated to save the computational time [23]. Since helium is a diatomic gas, the thermophysical properties of the working fluid at high-pressure and cryogenic
Fig. 1 Schematic diagram of modified Claude cycle-based helium liquefaction system

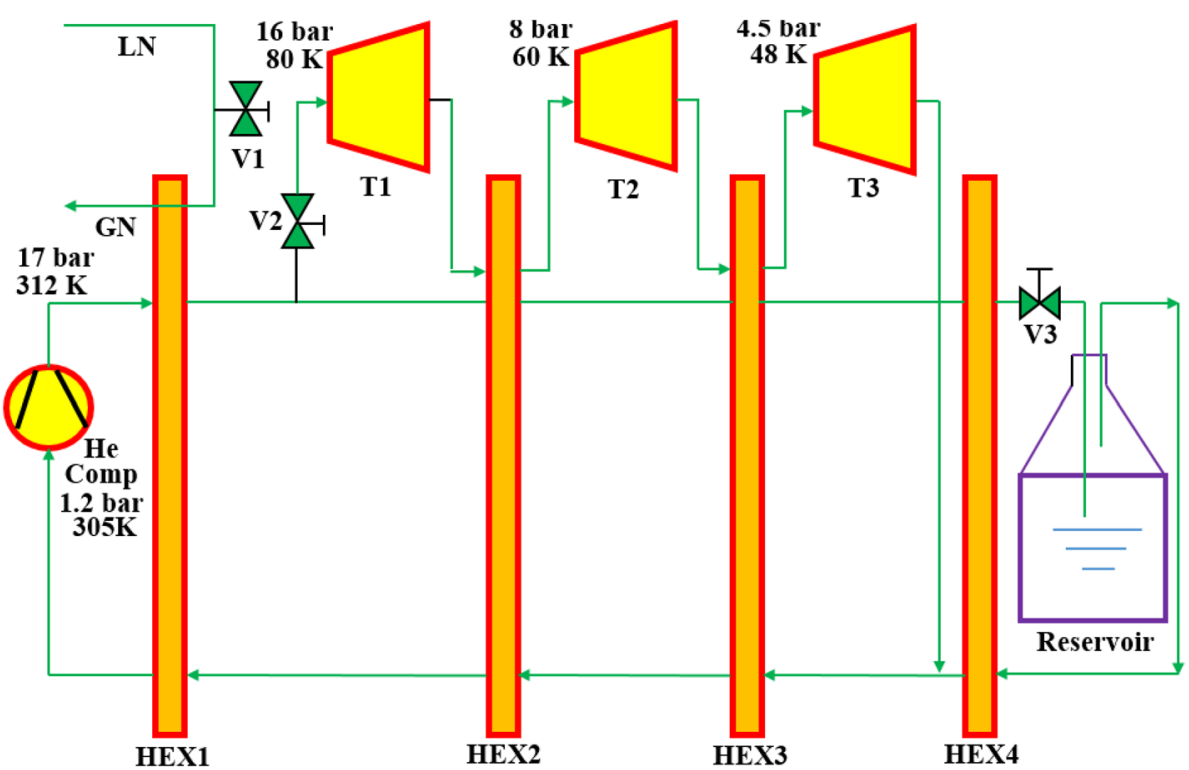

SN Applied Sciences A SPRINGER NATURE journa 
temperature have a remarkable effect on the aerodynamic design of the radial inflow turbine [24]. It is also necessary to select the real gas model to obtain the accurate design of the turbine blade profile. The inlet pressure, mass flow rate, expansion ratio, rotational speed, blade speed ratio, flow angles, and a number of blades, etc., are the aerodynamic decision variables on which the performance of the turbine depends. To maintain the flow in the subsonic region, stage loading $(\psi)$, and flow coefficient $(\phi)$ values are taken to be higher. To consider the machining precisions, low values of the degree of reaction $(\Omega)$ are taken, but in case of energy conversion in a single stage, its value should be higher. Therefore, it is a critical parameter, and its accurate range must be predicted carefully. Velocity ratio $\left(v_{s}\right)$, turbine diameter ratio $(D)$, and the number of blades $(Z)$ are other parameters on which the feasibility of the turbine design depends. The developed MATLAB ^ code is used for radial turbine design, and the thermophysical data are obtained from NIST's REFPROP'.

Figure 2 represents the mean-line design approach of a radial inflow turbine. The two important non-dimension parameters: specific speed and specific diameter, are used to determine the major dimensions of the turbine. The values are selected using Balje chart to get the maximum possible efficiency, and the fluid at the turbine inlet is in the subsonic regime. The inlet and exit velocity triangles of the turbine depend on these parameters.

- Specific speed

$$
n_{\mathrm{s}}=\frac{w \times \sqrt{Q_{3}}}{\left(\Delta h_{1-3, s}\right)^{3 / 4}}
$$

- Specific diameter

$$
d_{\mathrm{s}}=\frac{D_{2} \times\left(\Delta h_{1-3, s}\right) 1 / 4}{\sqrt{Q_{3}}}
$$

where $Q_{3}$ is the volumetric flow rate at the exit of the turbine and $\Delta h_{3, s}$ is the isentropic enthalpy drop from inlet to the turbine exit.

- Flow coefficient

$$
\phi=\frac{C_{m 3}}{U_{2}}=\frac{C_{m 2}}{\chi U_{2}}
$$

- Static head coefficient

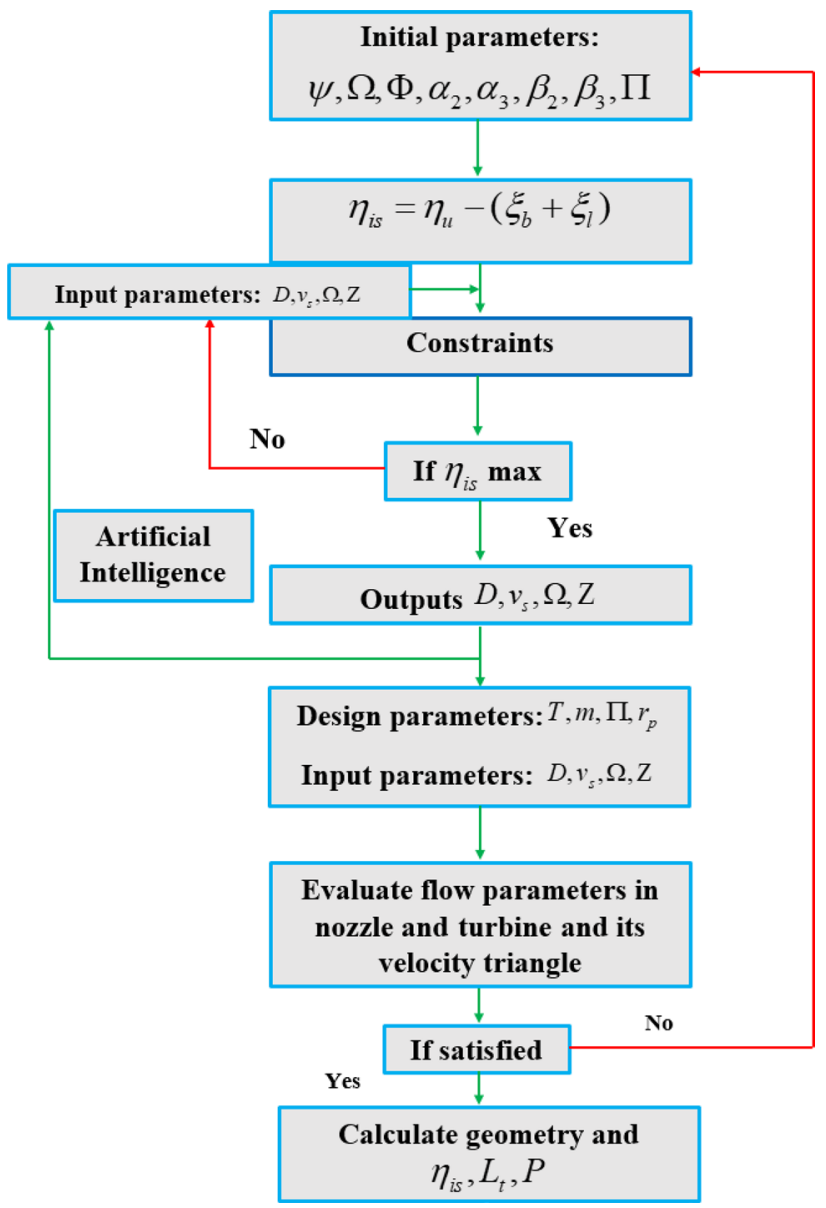

Fig. 2 Mean-line design approach of a radial inflow turbine

$$
\psi=\frac{\triangle h_{1-3}}{U_{2}^{2}}=\frac{C_{\theta 2}}{U_{2}}-\lambda \frac{C_{\theta 3}}{U_{2}}
$$

where $\chi$ represents the absolute meridional velocity at the inlet and outlet of the rotor and $\lambda$ is the outlet to inlet radius ratio of the turbine.

$\eta_{\text {is }}=\frac{h_{\text {in }}-h_{\text {ex }}}{h_{\text {in }}-h_{\mathrm{ex}, \mathrm{s}}}$

Power output,

$P=\dot{m}\left(h_{\text {in }}-h_{\text {ex }}\right)$

The velocity triangles and geometrical specifications of a radial inflow turbine are represented in Fig. 3.

\subsection{Reasonable non-dimensional variables and its sensitivity analysis}

It is observed from the mean-line design process that the isentropic efficiency, total losses, and power output of the 
Fig. 3 a The schematic diagram of the velocity triangle of the turbine. $\mathbf{b}$ The turbine specifications
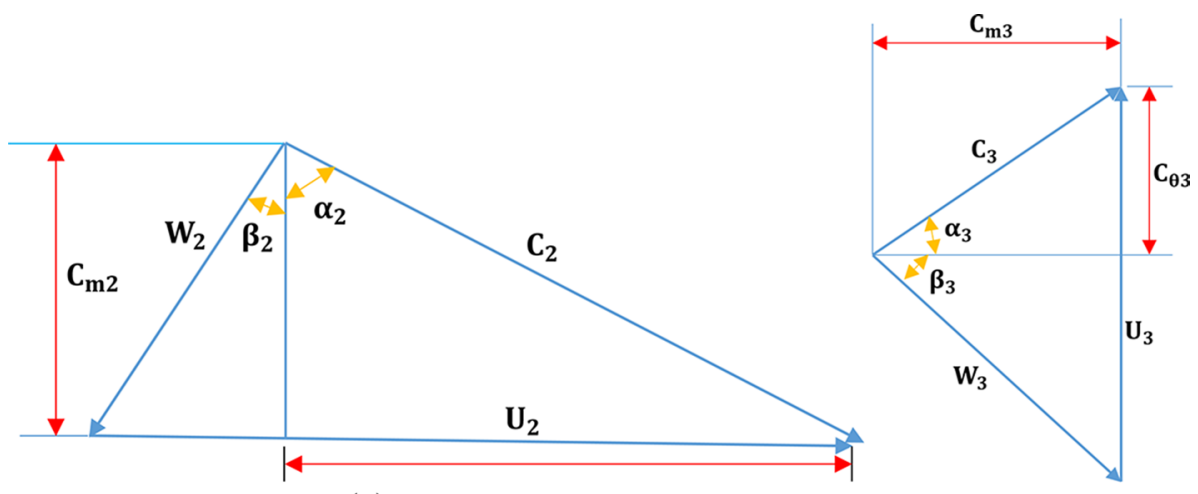

(a) Inlet and outlet velocity triangle of the turbine

$\mathbf{Z}_{\mathbf{r}}$

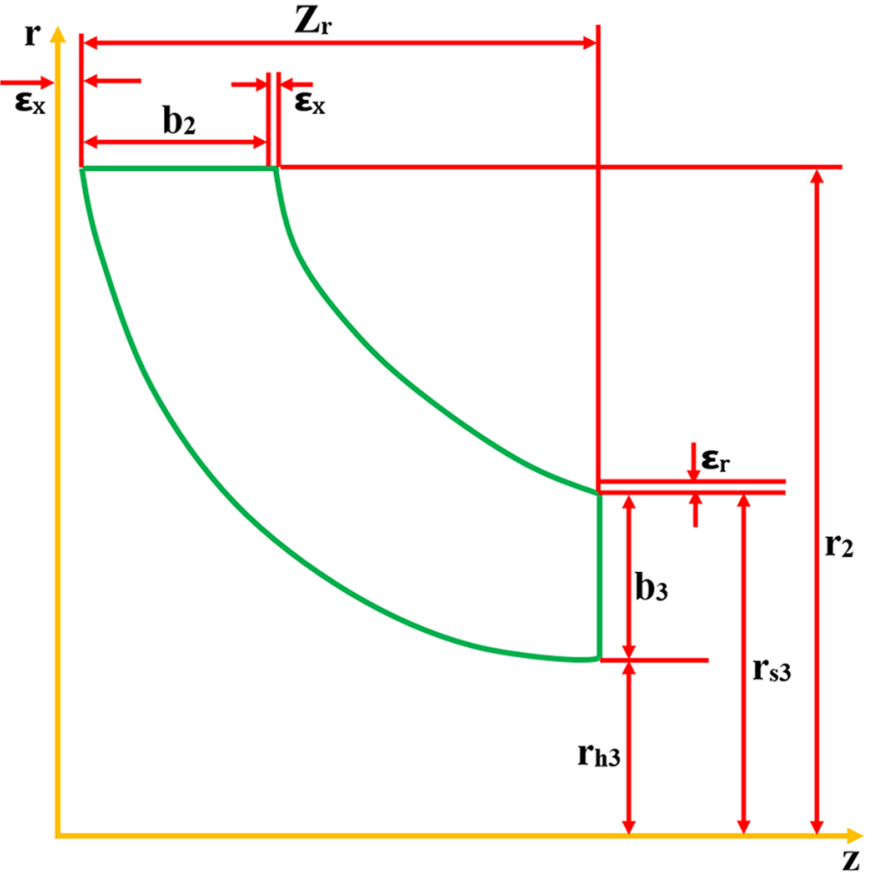

(b) turbine are significantly affected by the velocity ratio $\left(v_{s}\right)$, degree of reaction $(\Omega)$, nozzle velocity coefficient $(\phi)$, turbine velocity coefficient $(\psi)$, turbine diameter ratio $(D)$, velocity ratio $\left(v_{s}\right)$, absolute inlet flow angle $\left(\alpha_{1}\right)$, and relative outlet velocity angle $\left(\beta_{2}\right)$. The velocity components are important to encounter the flow losses. It depends on different parameters like wall friction, boundary layer separation, secondary flow, etc. Since the flow field inside the turbine is complex due to the rotation, the turbine velocity coefficient $(\psi)$ is usually less than the nozzle velocity coefficient $(\phi)$. The decrease in $\alpha_{1}$ increases the turbine efficiency, but at the same time, the nozzle losses may be increased. The decrease in $\beta_{2}$ reduces the turbine outlet velocity losses, but it is limited by the manufacturing constraint. The velocity ratio directly affects the efficiency of the turbine. The turbine diameter ratio $(D)$ is also an important parameter to define. The increase in diameter ratio increases the isentropic efficiency and reduces the total losses of the turbine. It is also useful in determining the outlet diameter of the turbine which is directly related to the impeller losses. The number of blades is a fundamental parameter on which the turbine losses and its efficiency depend. A lesser number of blades are always beneficial for flow stability, but the power output is low, whereas flow blockage and total losses are increased by increasing the number of blades. The most important correlations are identical to Zweifel's criterion to identify the number of blades in the turbine, but it is generally used for axial flow turbines and large size rotors. For small radial turbine operating at high-pressure and cryogenic temperature, the prediction of the optimal range of the number of blades is necessary. To overcome the inertia of the blade, low entropy generation, and flow stability at the outlet of the turbine, the concept of splitter blades is also introduced. The number of main and splitter blades is determined based on the correlation proposed by Glassman [25] which is as follows: 
$Z=\frac{\pi}{30}\left(110-\alpha_{1}\right) \tan \alpha_{1}$

where $\alpha=90-\alpha_{2}$.

\subsubsection{Sobol method}

The sensitivity of different non-dimensional design variables on isentropic efficiency of the radial turbine is obtained from Sobol sensitivity analysis based on our previous work [4].

The variance decomposition, the total output variance to input constants are derived as follows:

$\operatorname{Var}(Y)=\sum D_{i}(Y)+\sum D_{i j}(Y)+\cdots+D_{12 \ldots d}(Y)$

where $\operatorname{Var}(Y)$ is the total variance of total loss (output), $D_{i}(Y)$ is the first-order variance of input constants $X_{i}$, and $D_{i j}(Y)$ is the second-order variance of $X_{i}$ and $X_{j}$ which are formulated as:

$\operatorname{Var}(Y)=E\left(Y_{i}^{2}\right)-E^{2}\left(Y_{i}\right)$

$D_{i}(Y)=\operatorname{Var}\left[E\left(Y \mid X_{i}\right)\right]$

Thus, the first-order sensitivity index is written as follows:

$S_{i}=\frac{D_{i}(Y)}{\operatorname{Var}(Y)}$

Figure 4 illustrates the normalized sensitivity index of six non-dimensional design variables. It is found out that the variation of isentropic efficiency is higher for $D, v_{s}, \Omega$, and $Z$ as compared to $\phi$ and $\psi$. Therefore, other variables are

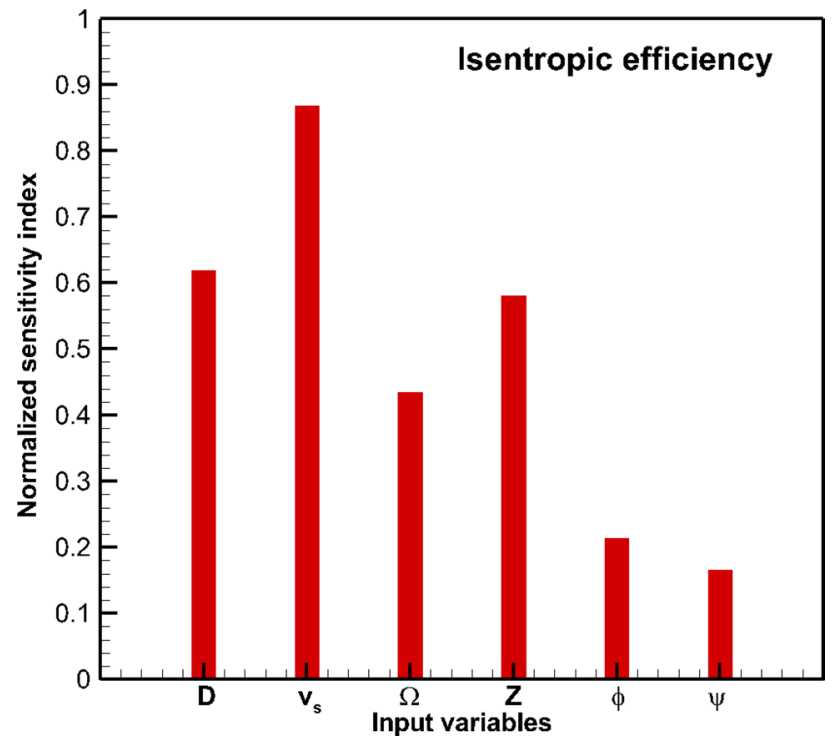

Fig. 4 Sensitivity analysis of isentropic efficiency determined first within the specified range after that the optimal range of these four variables is obtained.

Finally, the isentropic efficiency, total losses, and power output are defined in the functional form as follows:

$\eta_{\text {is }}=f\left(v_{s}, \Omega, D, Z\right)$

$L_{\mathrm{t}}=f\left(v_{\mathrm{s}}, \Omega, D, Z\right)$

$P=f\left(v_{s}, \Omega, D, Z\right)$

\section{ANN and ANFIS model development}

The artificial neural network (ANN) and adaptive neuro-fuzzy inference system (ANFIS) are soft computing tools which are used to predict the optimal range of design variables of a radial inflow turbine. The models are developed to predict the isentropic efficiency, total losses, and power output of a radial inflow turbine with a set of design variables using $M A T L A B \wedge 17 . b$ toolbox.

\subsection{Artificial neural networks}

The ANN is an information processing tool based on the biological neurons and a function-based approach. The neural network has a huge number of interconnected artificial neurons (nodes or processing elements) operated in parallel. These nodes are renowned as bias neurons. It has an input layer, one or more hidden layers, and an output layer. The neurons are the heart of the neural network. Generally, an additional neuron is added in hidden and output layers. The neuron in this layer gets their input information from the output neurons of the previous layer. Every neuron is incorporated with a transfer function which determines the output by summing up the intermediate neurons in the hidden and output layers.

The neural network obtains knowledge during the training process which is generally stored in between the neurons also known as synaptic weights. It can be adjusted according to supervised and unsupervised training. In the supervised training, the neural network learns from labeled training datasets of existing examples, whereas for unsupervised training, the network learns from unlabeled training datasets of existing examples. The multi-layer perceptron (MLP) is the most popular neural network model which is trained by the backpropagation algorithm. It can reduce the error between the desired output value and the corresponding output value by adjusting the synaptic weights. The neural network can learn both linear and nonlinear relationships directly.

Since the neural network has no explicit rules, it is fully dependent on the training datasets. It is helpful in applications of the complex problems in which the traditional 
modeling methods are not appropriate to simulate the system. It has several inputs $\left(x_{i}\right)$. Every input multiplies with their corresponding weight $\left(w_{i}\right)$ and then summed up. The results are obtained in terms of neuron's activation function $z$ as follows:

$z=\sum_{i=1}^{p} w_{i} x_{i}+b$

where $b$ is the bias. Once the $z$ is obtained, it is used as an activation function $f(z)$. The output of these activation functions is the neuron output.

Generally, the neurons are gathered in the form of layers. These layers are of three types:

Layer1 It receives the datasets from the outside and also known as the input layer.
Layer2 The hidden layers for which the input and output datasets are inside the network.

Layer3 The output layer of the network.

The feed-forward network is used in which the neurons of each layer are connected to the very next layer.

\subsection{Neural network learning}

The training of ANN is a procedure to minimize the error function between the input and output by adjusting the weights and biases of a network. A gradient descent backpropagation procedure is used during the training. For selecting the best backpropagation algorithm, five backpropagation algorithms are opted to predict the best linear equation, RMSE error, and $R^{2}$ for isentropic efficiency of the turbine. Additionally, two neurons are used in the hidden layer for all the backpropagation algorithms. Table 1

Table 1 Comparison of 5 backpropagation algorithms with two neurons in the hidden layer

\begin{tabular}{lllllr}
\hline Backpropagation algorithm & Function & RMSE & Epoch & $\begin{array}{l}\text { Correlation coef- Best linear equation } \\
\text { ficient }\left(R^{2}\right)\end{array}$ \\
\hline Levenberg-Marquardt backpropagation & & & & & \\
Batch gradient descent & Trainlm & 0.00235005 & 200 & 0.974 & $Y=0.970 X+0.0064$ \\
Batch gradient descent with momentum & Trainlm & 0.02753381 & 200 & 0.965 & $Y=0.956 X+0.027$ \\
Scaled conjugate gradient backpropagation & Trainlm & 0.50820237 & 200 & 0.887 & $Y=0.88 X+0.137$ \\
Fletcher-Reeves conjugate gradient backpropagation & Trainlm & 0.44944913 & 200 & 0.821 & $Y=0.81 X+0.183$ \\
& Trainlm & 0.48619630 & 200 & 0.782 & $Y=0.769 X+0.148$ \\
\hline
\end{tabular}

Fig. 5 Schematic of the ANN model network
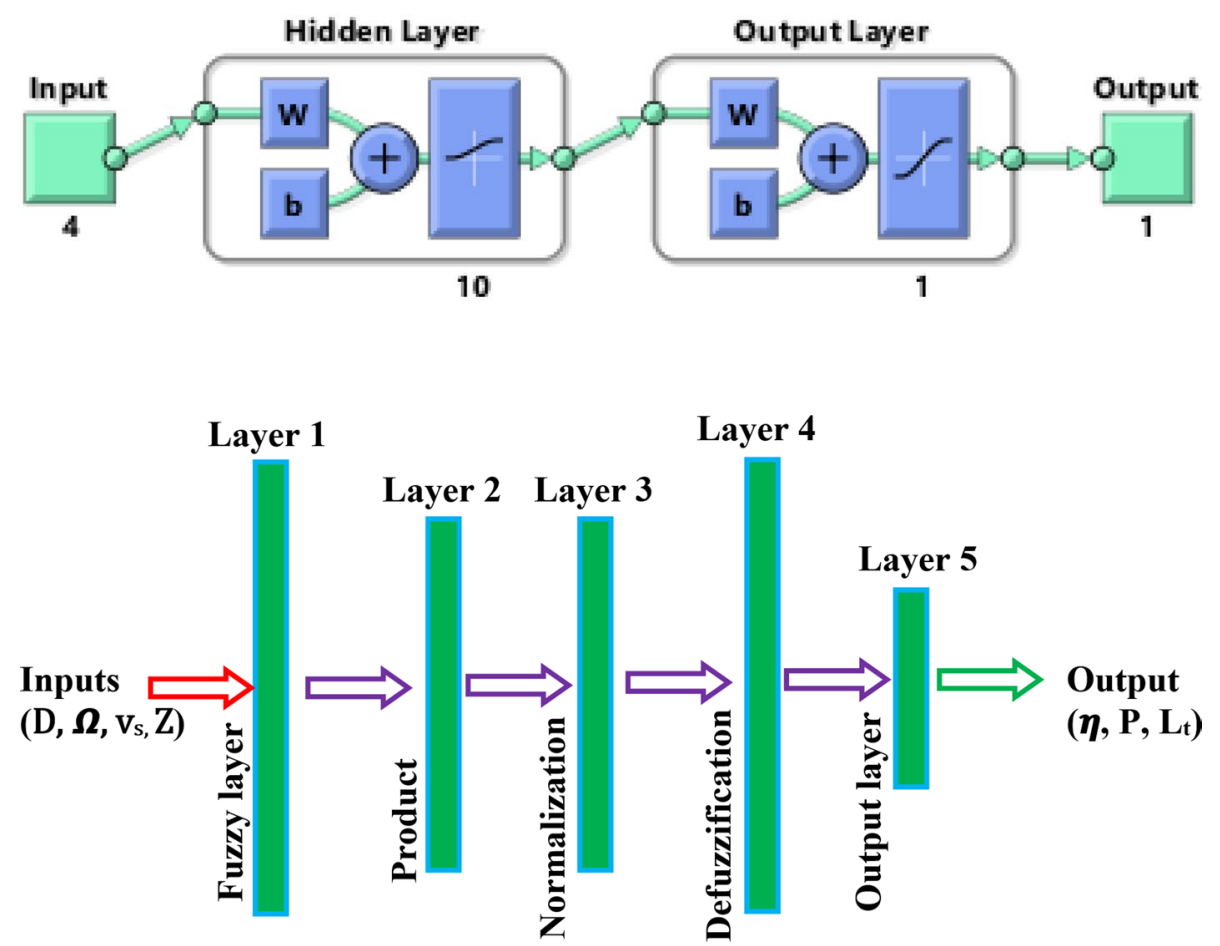

Fig. 6 The basic structure of ANFIS 


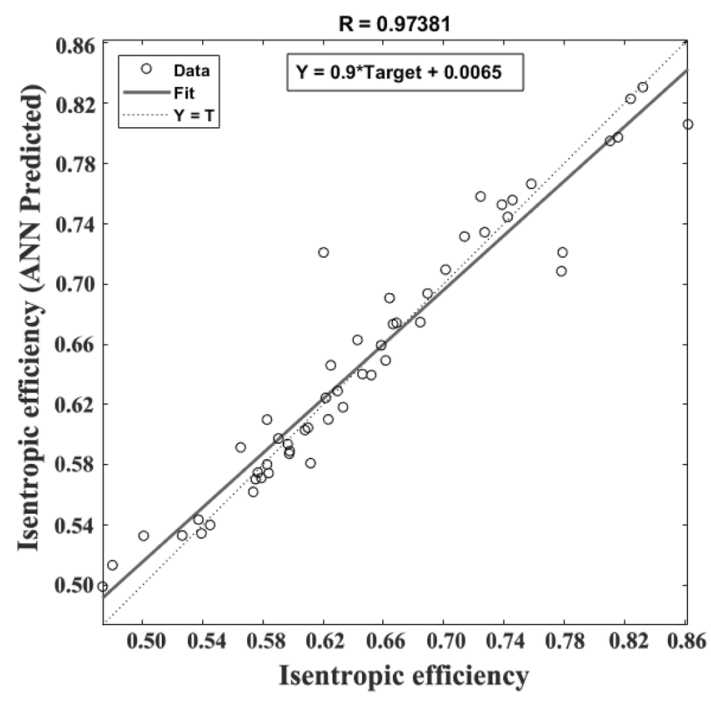

(a)

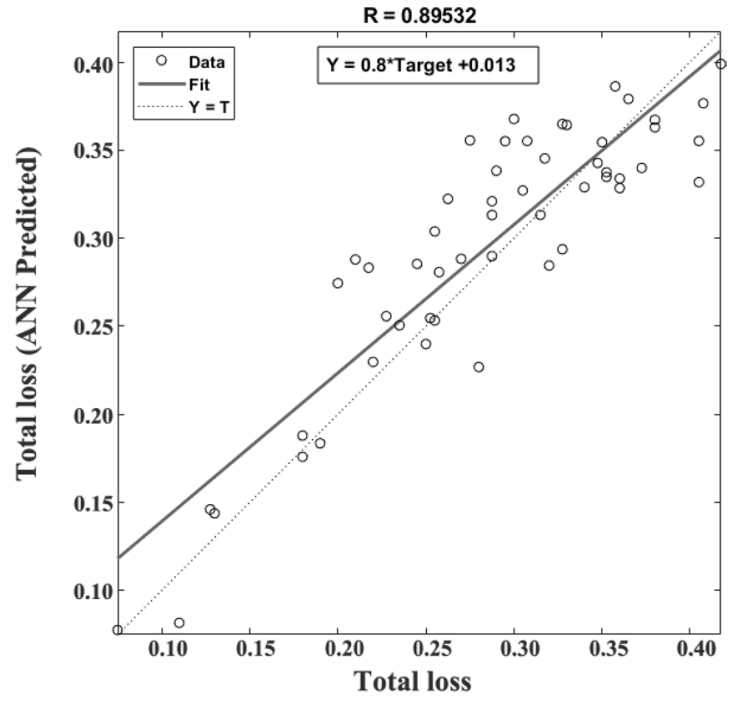

(b)

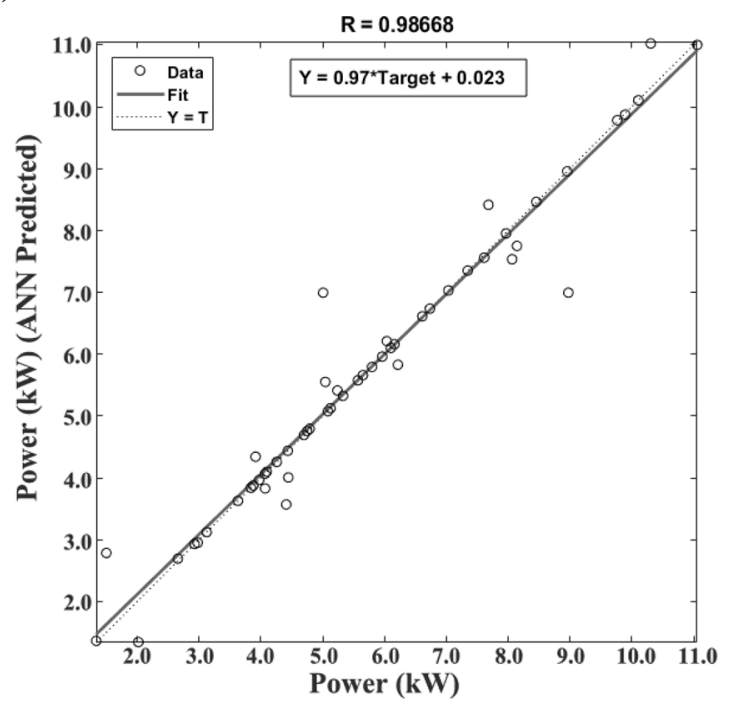

(c)

Fig. 7 Regression curve of ANN. a Isentropic efficiency $\eta_{\text {is }}$. b Total losses $\left(L_{t}\right)$. c Power output

shows a comparison of different backpropagation training algorithms. Among these, the Levenberg-Marquardt backpropagation training algorithm has the least mean square error and faster training as compared to other algorithms because it is second-order accurate and cannot compute the Hessian matrix. The performance of the ANN network is computed by the root mean square error (RMSE) and regression coefficient $\left(R^{2}\right)$ for the accuracy of the model and obtained from the equations as follows:

RMSE $=\sqrt{\frac{\sum_{n=1}^{N}\left(y_{n, \text { pred }}-y_{n, \text { exp }}\right)^{2}}{N}}$

$$
R^{2}=1-\frac{\sum_{n=1}^{N}\left(y_{n, \text { pred }}-y_{n, \exp }\right)^{2}}{\sum_{n=1}^{N}\left(y_{n, \exp }-y_{m}\right)^{2}}
$$

where $N$ is the number of data points, $y_{n, \text { pred }}$ is the network prediction, $y_{n, \text { exp }}$ is the actual response, $y_{m}$ is the average of actual values, and $n$ is an index of data. Subsequently, RMSE and $R^{2}$ are computed to check the performance of the network. The minimum value provides the best ANN network. 
Fig. 8 Decision surface of isentropic efficiency a $\Omega$ and $Z$, b $v_{s}$ and $D$

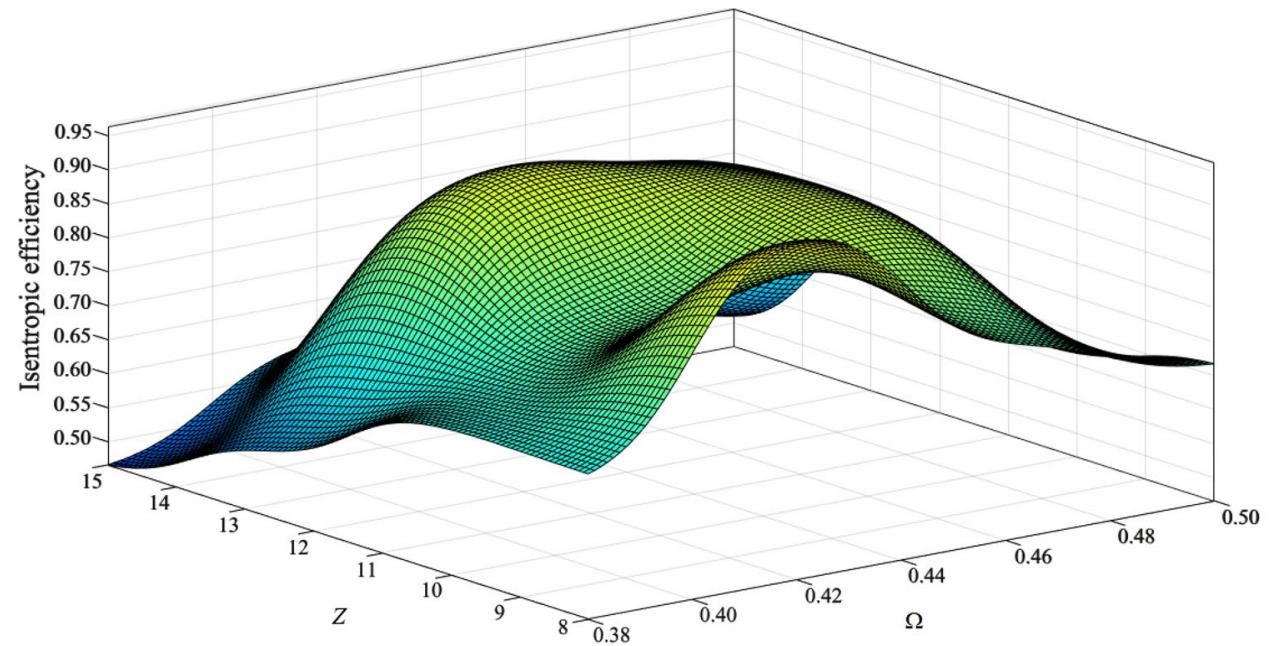

(a)

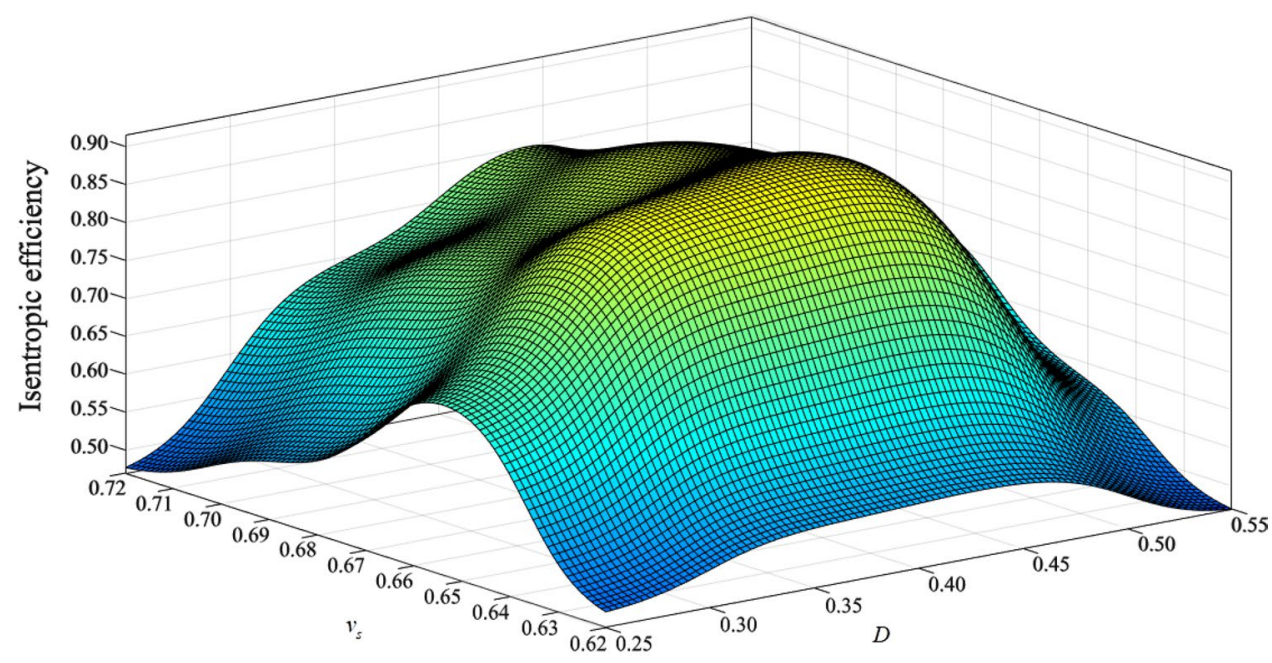

(b)

\subsection{Neural network training and testing}

The input and target data are selected based on the design variables on which the performance of the turbine is profoundly affected. The feed-forward network with one hidden layer is selected in the present study. Therefore, it is a three-layer network including the input and output layer as represented in Fig. 5.

Literature review suggests that the single hidden layer is enough for 'universal approximation' property, but it requires a higher number of neurons. Since the addition of the second hidden layer provides better results with lesser weights, it is beneficial where the input and output datasets are more, and the functions have a large peak and valley. Additionally, the supervised learning process is considered for those cases [26]. If the datasets are less $(<100)$, it is recommended to use single hidden layer [4]. In the present study, Logsig, Purelin, and Tansig transfer functions are used, and the predicted results are compared.

Several configurations of ANN and epochs are endeavored to minimize the mean square error (MSE).

$\mathrm{MSE}=\frac{1}{n_{\mathrm{T}} n_{\mathrm{P}}} \sum_{j=1}^{n_{\mathrm{T}}} \sum_{i=1}^{n_{\mathrm{P}}}\left(T_{i j}-S_{i j}\right)^{2}$

where $n_{\mathrm{T}}$ and $n_{\mathrm{P}}$ are the number of targets and patterns, respectively. $T_{i j}$ and $S_{i j}$ represent the target and simulated vectors, respectively. 
Fig. 9 Decision surface of total losses $\mathbf{a} \Omega$ and $Z \mathbf{b} v_{\mathrm{s}}$ and $\mathrm{D}$

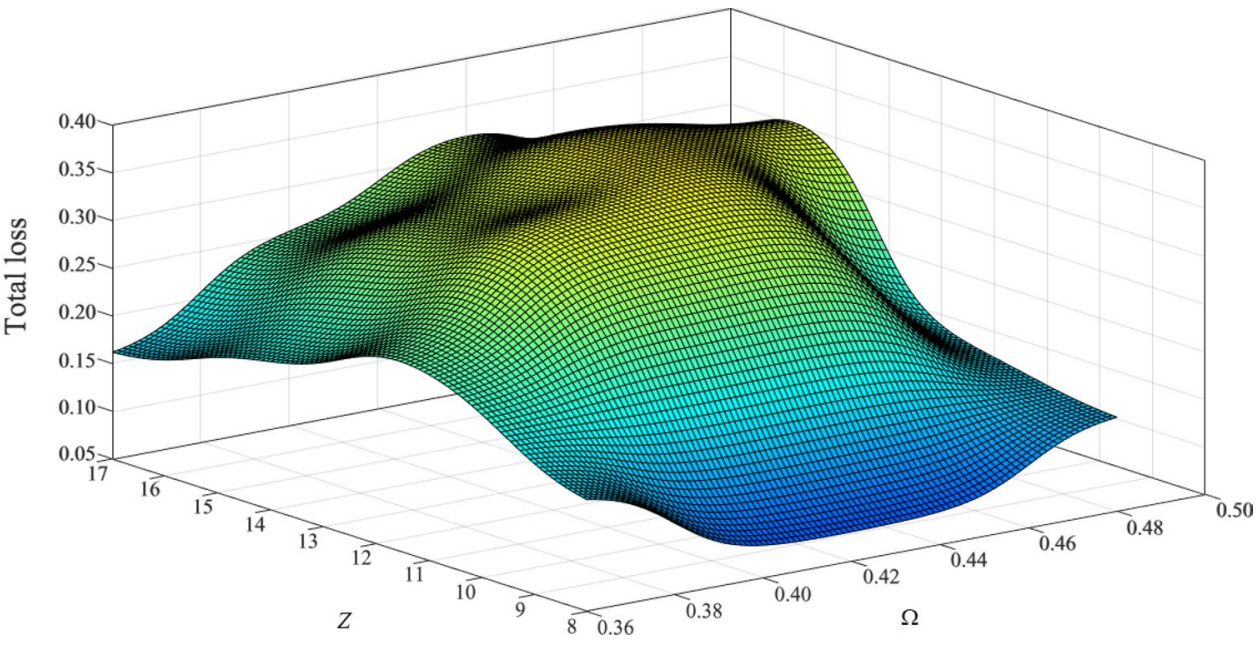

(a)

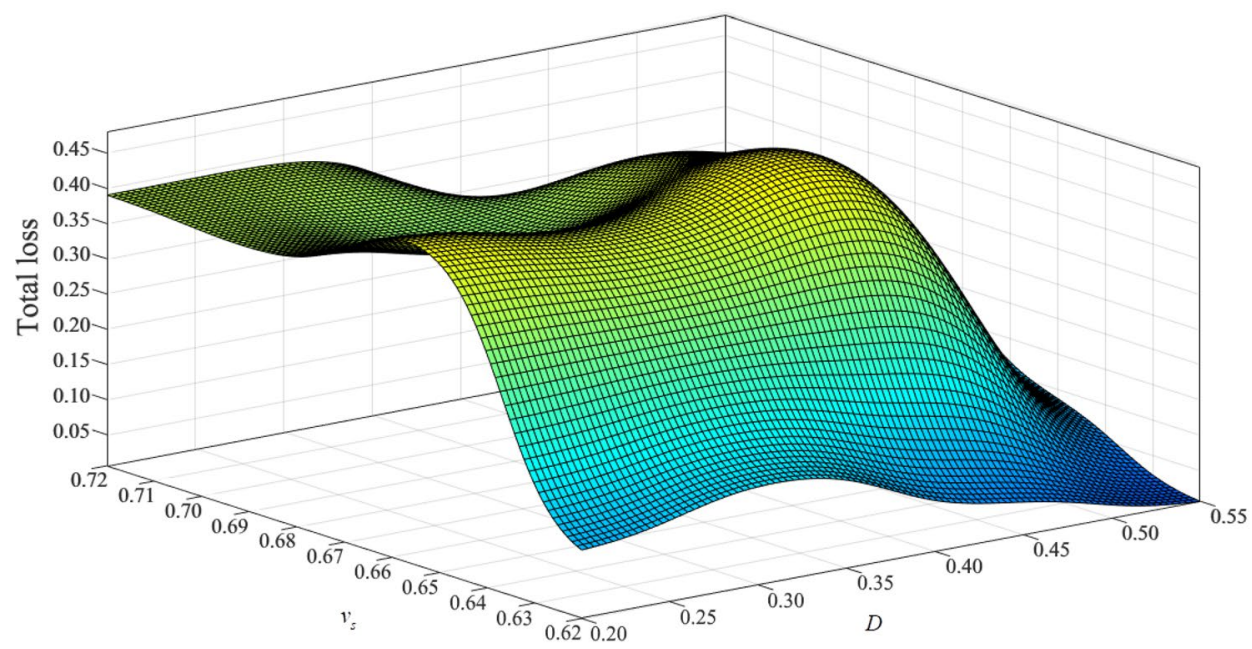

(b)
The network is trained using backpropagation gradient descent algorithm. The iterations are carried out by varying the weights and step size to obtain the global minima. When higher learning rate is opted, the convergence gets faster, but it leads to instability of the network, and consequently, the errors increase. Therefore, for stable learning, the learning rate is limited to the inverse of the largest eigenvalue of the correlation matrix of the input datasets.

\subsection{ANFIS model development}

The fuzzy system is a computing framework originated from fuzzy set theory, if-then rules, and fuzzy reasoning. When the system combines with the neural network, it advantages the assimilating features of the neural network and has an implementation equal to the fuzzy inference model [27]. The fuzzy logic plays a significant role in the design and development of a network which predicts the optimal range of design variables for the best performance of the radial turbine. In this regard, the selection of membership function (MF) type, a number of rules, and selection of input and output variables are decision-making process which needs specialized knowledge and very much important for desired output.

Adaptive neuro-fuzzy inference system is the amalgamation of ANN and fuzzy inference systems. Since the ANFIS is the combination of neural network and fuzzy inference system, the neuro-fuzzy approach quantifies the fuzzy system by using neural network methods. In the adaptive neuro-fuzzy model, two learning algorithms are needed as follows:

- Structural learning algorithm to obtain satisfactory fuzzy rules. 
Fig. 10 Decision surface of power output a $\Omega$ and Z, $\mathbf{b} v_{s}$ and D
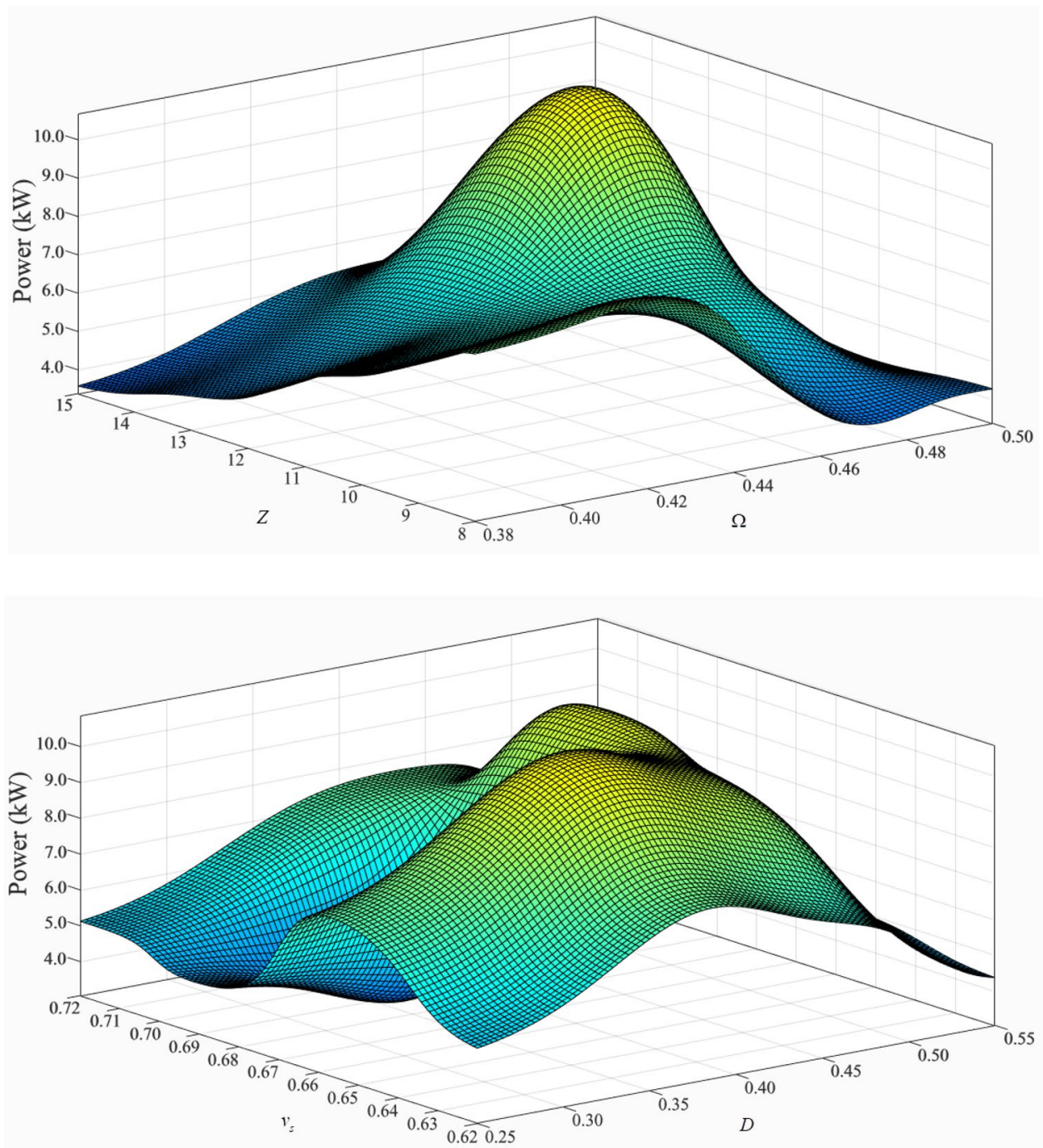

(b)
- Parameter learning algorithm to modify the MFs and other parameters according to the better prediction capability.

In the present study, the gradient descent training algorithms are used to get fuzzy system parameters. The ANFIS structure is expressed by two if-then rules derived from Takagi-Sugeno $(T-S)$ model as follows:

Rule1 If ( $\mathrm{x}$ is $\left.A_{1}\right)$ and ( $\mathrm{y}$ is $B_{1}$ ) then $f_{1}=p_{1} \mathrm{x}+q_{1} \mathrm{y}+r_{1}$ Rule2 If $\left(\mathrm{x}\right.$ is $\left.A_{2}\right)$ and $\left(\mathrm{y}\right.$ is $\left.B_{2}\right)$ then $f_{2}=p_{2} x+q_{2} y+r_{2}$

where $p_{i}, r_{i}$, and $q_{i}$ are the design parameters which are determined during the training process. The ANFIS system has $T-S$ rules in five layers using a multi-iteration learning procedure and hybrid learning algorithm. The first layer has input variables (MFs). This layer has three adaptable parameters which are linked to input MFs $\left(a_{i}, b_{i}\right.$, and $\left.c_{i}\right)$ and endows the input values to the very next layer. The second layer (membership layer) examines the weights of the MFs. The upcoming values from the previous layer act as MFs for this layer to represent the fuzzy sets of the input variables. However, the neurons are non-adaptive in this layer which can multiply the incoming signals. The output of each neuron shows the firing strength of the rule.

The third layer is also renowned as the fuzzy rule layer which is non-adaptive. In this layer, the activation level is computed for each rule. Generally, the number of layers is equal to the number of fuzzy rules. The weights of these layers are normalized by calculating the ratio of the firing strength rule to the sum of firing strengths of all the rules as mentioned. The fourth layer is the defuzzification layer which produces the output values due to an inference of rules. This layer has three adaptable parameters $\left(p_{i}, r_{i}\right.$, and $q_{i}$ ) which are first-order polynomials and called as the result parameter. The nodes are adaptive, and the node function 


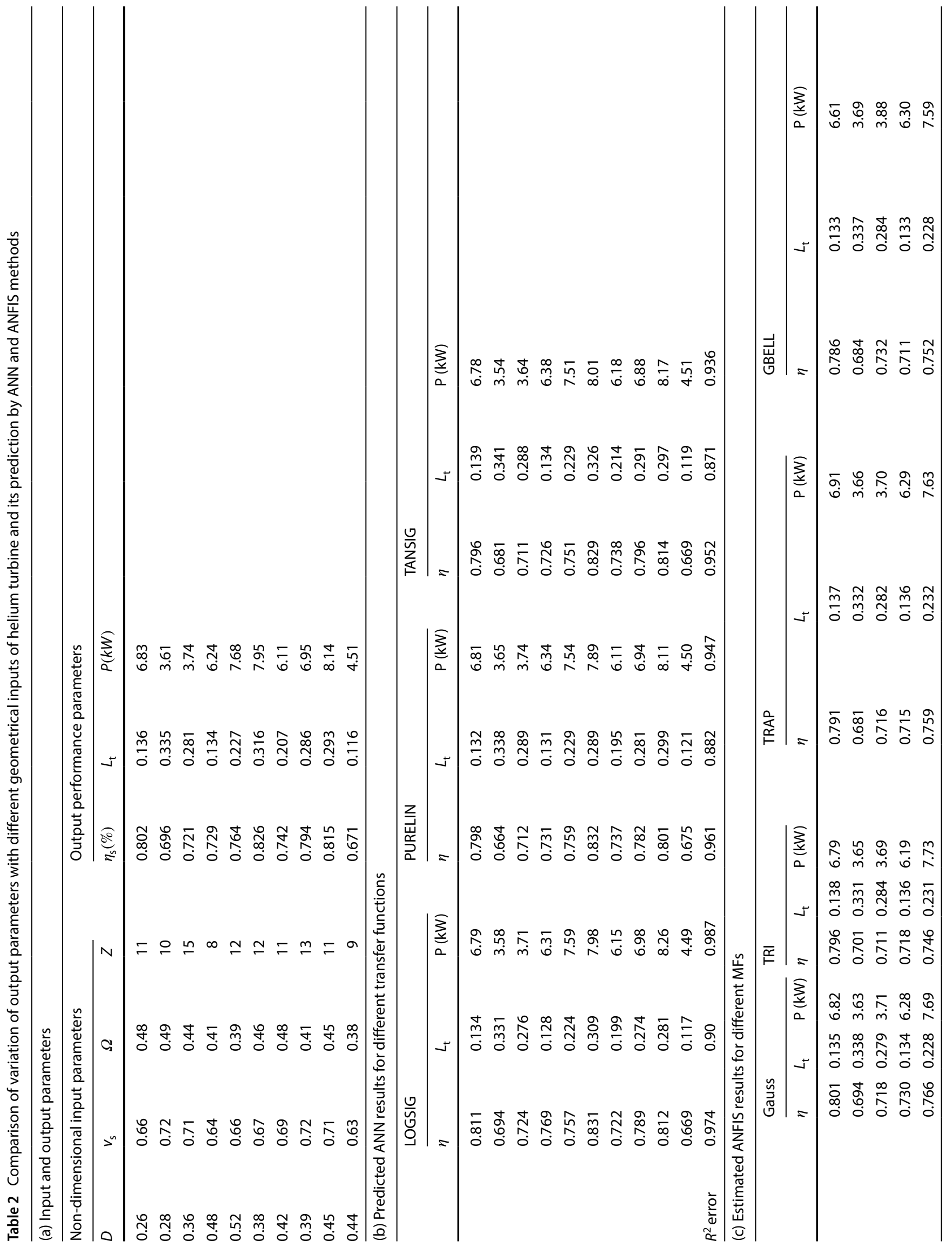




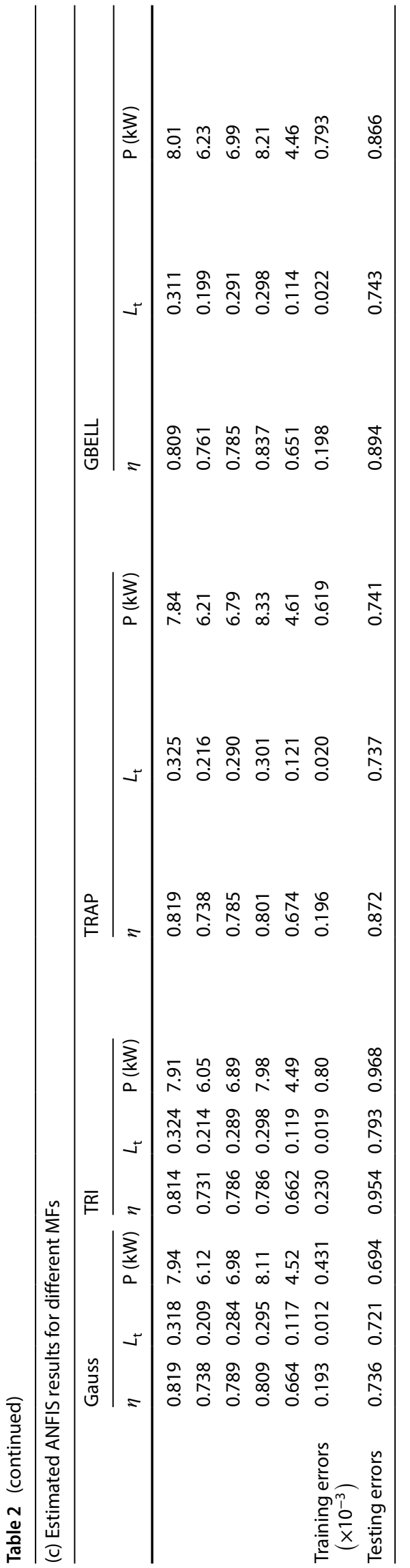

is known as the result parameters. The fifth layer is nonadaptive which summed up all the incoming signals and converted it into one single output.

The learning or training algorithm can change the adaptable parameters to compare the ANFIS outputs. $a_{i}, b_{i}$, and $c_{i}$ are the $M F$ parameters which define their centers. The training period is divided into two segments. The first one adjusts the result parameters with the least-squares method, and the second one is adaptable with gradient descent (backpropagation) method. Conclusively, the ANFIS output is expressed by the correlation mentioned as follows:

$$
\begin{aligned}
& z=\frac{w_{1}}{w_{1}+w_{2}} z_{1}+\frac{w_{2}}{w_{1}+w_{2}} z_{2} \\
& =\bar{w}_{1}\left(p_{1} x+q_{1} y+r_{1}\right)+\bar{w}_{2}\left(p_{2} x+q_{2} y+r_{2}\right) \\
& =\left(\overline{w_{1} x}\right) p_{1}+\left(\overline{\mathbf{w}_{\mathbf{1}} \mathbf{y}}\right) \mathbf{q}_{\mathbf{1}}+\left(\overline{\mathbf{w}_{\mathbf{1}}}\right) \mathbf{r}_{\mathbf{1}} \\
& +\left(\overline{w_{2} x}\right) p_{2}+\left(\overline{w_{2} y}\right) q_{2}+\left(\overline{w_{2}}\right) r_{2}
\end{aligned}
$$

Equation 19 shows that the output ( $z$ ) of Sugeno Fuzzy system is a linear combination of adaptable parameters. Therefore, an amalgamation of gradient descent and least-square methods can characterize the optimal values of the output parameters ( $p, q$, and $r$ ). However, if the $M F$ changes, the trained area becomes wider, and convergence may get slower. For such cases, the hybrid learning algorithm with gradient descent and least-square methods produces better results.

The performance of a radial turbine can be accurately predicted by selecting the more appropriate input parameters. The adequate contemplation of all the decision variables is crucial in deciding and developing a reliable ANFIS model. In this regard, the non-dimensional variables $(D, \phi$, $\Omega$, and $v_{s}$ ) used during the design process are considered as an input parameter in the training process. The output parameters are isentropic efficiency $\left(\eta_{\text {is }}\right)$, total losses $\left(L_{\mathrm{t}}\right)$, and power output. These datasets are obtained from the design procedure of the radial inflow turbine. There are four input and three output variables which are divided into $80 \%$ and $20 \%$ datasets for training and validation purpose.

\subsection{Structure of adaptive neuro-fuzzy inference system}

Membership functions (MFs) are determined to establish an ANFIS network. MFs are arbitrary curves which depend on the type of application that can be used. There are various membership functions available, but for the present case, Gauss, Tri, Trap, and Gbell MFs are employed for input parameters and compare their predicted results, 
Table 3 Major geometrical specifications of the turbine and nozzle

\begin{tabular}{|c|c|c|c|}
\hline Specifications & High-pressure & Medium pressure & Low pressure \\
\hline Turbine diameter (mm) & 15.74 & 16.08 & 16.22 \\
\hline Turbine inlet blade height (mm) & 0.65 & 0.67 & 0.68 \\
\hline Turbine outlet blade height $(\mathrm{mm})$ & 2.65 & 2.68 & 2.71 \\
\hline Turbine hub diameter (mm) & 5.21 & 5.24 & 5.25 \\
\hline Shroud clearance (mm) & 0.1 & 0.1 & 0.1 \\
\hline Number of nozzle blades & 15 & 17 & 19 \\
\hline Number of turbine blades & 7 & 8 & 9 \\
\hline Number of splitter blades & 7 & 8 & 9 \\
\hline Tip clearance (mm) & 0.1 & 0.1 & 0.1 \\
\hline
\end{tabular}

Table 4 Node distribution and its scheme in different blocks

\begin{tabular}{|c|c|c|c|c|c|c|}
\hline \multirow{2}{*}{$\begin{array}{l}\text { (High/medium/low } \\
\text { pressure) }\end{array}$} & \multicolumn{2}{|l|}{ Inlet block } & \multicolumn{2}{|l|}{ Outlet block } & \multicolumn{2}{|c|}{ Passage block } \\
\hline & Streamwise & Blade-to-blade & Streamwise & Blade-to-blade & Streamwise & Blade-to-blade \\
\hline Turbine & $22 / 20 / 20$ & $54 / 52 / 50$ & $20 / 20 / 18$ & $36 / 34 / 34$ & $86 / 86 / 82$ & $40 / 40 / 38$ \\
\hline Nozzle & $16 / 16 / 16$ & $35 / 36 / 36$ & $14 / 14 / 14$ & $34 / 32 / 34$ & $54 / 54 / 50$ & $28 / 28 / 28$ \\
\hline
\end{tabular}

Table 5 Non-dimensional wall distance of the turbine and nozzle

\begin{tabular}{llll}
\hline Sections (high/medium/low pressure) & $y+$ value & Sections (high/medium/low pressure) & $y+$ value \\
\hline Turbine inlet & $0.8 / 0.8 / 0.8$ & Nozzle inlet & $0.89 / 0.88 / 0.88$ \\
Near turbine blade & $0.72 / 0.72 / 0.72$ & Near Nozzle blade & $0.84 / 0.84 / 0.82$ \\
Center of the flow passage & $1.2 / 1.2 / 1.3$ & Center of the flow passage & $1.3 / 1.3 / 1.4$ \\
Turbine outlet & $0.84 / 0.84 / 0.82$ & Nozzle outlet & $0.78 / 0.80 / 0.80$ \\
\hline
\end{tabular}

Fig. 11 Computational fluid domain and boundary set-up for nozzle and turbine

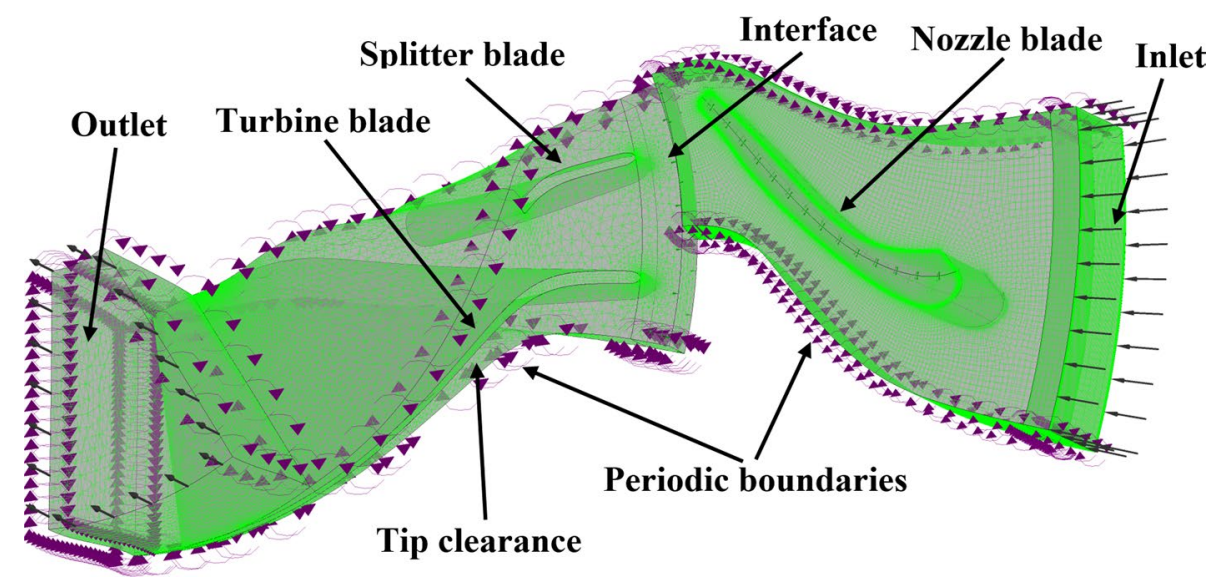

\begin{tabular}{lll}
\hline & Advection scheme & Transient scheme \\
\hline Continuity & Upwind & Second-order backward Euler \\
Momentum & Upwind & Second-order backward Euler \\
Energy & Upwind & Second-order backward Euler \\
Turbulent kinetic energy & High-resolution & High-resolution \\
Turbulent eddy frequency & High-resolution & High-resolution \\
\hline
\end{tabular}

Table 6 The discretization schemes for different governing equations
SN Applied Sciences 


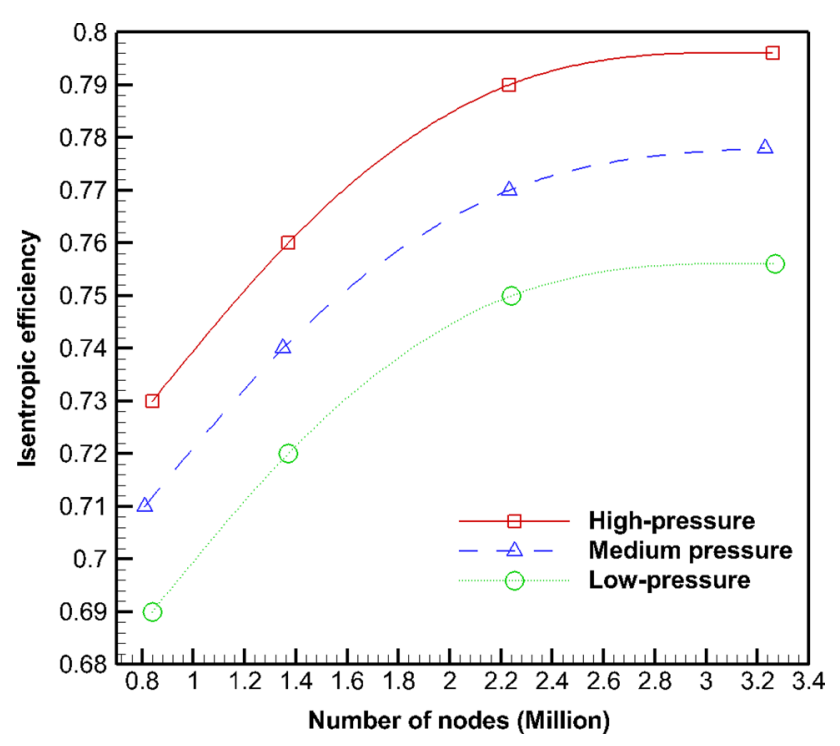

Fig. 12 Grid convergence study

training, and testing errors. For the output parameters, a hybrid function has opted. Theoretically, the increase of MFs can decrease the error until it reaches its minimum value after that further increase of MFs deduces higher errors and computational time. Therefore, to determine the optimum number of MFs, the error occurred from each network is checked by varying the number of MFs until the errors stop to decrease and that MFs are the optimum one. In the present case, four MFs for every input are opted [28]. The basic structural information of ANFIS network is shown in Fig. 6.

The performance of the model is verified using three error factors including R-squared, mean absolute error $(M A E)$, and root mean squared error (RMSE) which are as follows:

$R^{2}=1-\frac{\sum_{t=1}^{N}\left(f_{\mathrm{t}}-O_{\mathrm{t}}\right)^{2}}{\Sigma_{t=1}^{N}\left(f_{\mathrm{t}}-\bar{f}_{\mathrm{t}}\right)^{2}}$

$\mathrm{MAE}=\frac{1}{N} \times \Sigma_{t=1}^{N}\left|f_{\mathrm{t}}-O_{\mathrm{t}}\right|$

RMSE $=\sqrt{\frac{1}{N} \times \Sigma_{t=1}^{N}\left(f_{\mathrm{t}}-O_{\mathrm{t}}\right)^{2}}$

where $O_{\mathrm{t}}$ is model output, $f_{\mathrm{t}}$ is overall output, $N$ is total number of samples, and $t$ is number of samples.

\subsection{ANN and ANFIS results}

Table 2 represents that the errors obtained from LOGSIG transfer function (for ANN) are minimum. Therefore, the regression curves for LOGSIG TFs are presented. Figure 7 illustrates the comparison of regression curves of calculated and predicted isentropic efficiency, total losses, and power output. It depicts the developed ANN network is reliable. There are three networks: ANFIS-1 predicts isentropic efficiency, ANFIS-2 predicts the total losses, and ANFIS-3 predicts the power output. Four different type MFs (Gauss, Tri, Trap, and Gbell) are used during the training process, out of which Gauss MF has less error (Table 2c). Therefore, decision surfaces obtained from Gauss MF are presented hereafter. The final decision surfaces obtained from ANFIS training are represented in Figs. 8, 9, and 10. It illustrates that the isentropic efficiency of the turbine is higher when the degree of reaction and number of blades are in the range of $0.42-0.48$ and $10-14$, whereas the diameter ratio and velocity ratio are in the range of $0.35-0.50$ and $0.67-0.70$, respectively. In these ranges, the power output is on the higher side which is directly related to the refrigerating capacity of the system but total losses are slightly higher in these zones. The samples of non-dimensional input and output parameters and their predicted values from ANN and ANFIS are summarized in Table 2. Based on the predicted geometrical variables, the major geometrical specifications of the turbine and nozzle are mentioned in Table 3.

\section{Numerical methodology}

\subsection{Computational domain and mesh generation}

The turbine and nozzle dimensions obtained from the men-line design are exported to ANSYS BladeGen 18.1 to generate the three-dimensional model of the flow passage, inlet and outlet block. Thereafter, the computational domain is discretized using a multi-block structured grid using Turbo-Grid. The ATM optimized topology is opted to generate the three-dimensional mesh in the nozzle and turbine. The $\mathrm{H}$-type topology is used for inlet and outlet blocks, whereas O-type is opted for the passage block to solve the near-wall regions which are required for boundary layer flow visualization near the wall. The node distribution of the grid and its scheme are mentioned in Table 4. The O-grid includes in the periodic boundary and from hub to shroud in the passage block. Since the mesh in the nozzle and turbine is generated separately, the general grid interface (GGI) connection is opted to combine the interface mesh in ANSYS CFX. Apart from this, the $y+$ value, which shows 


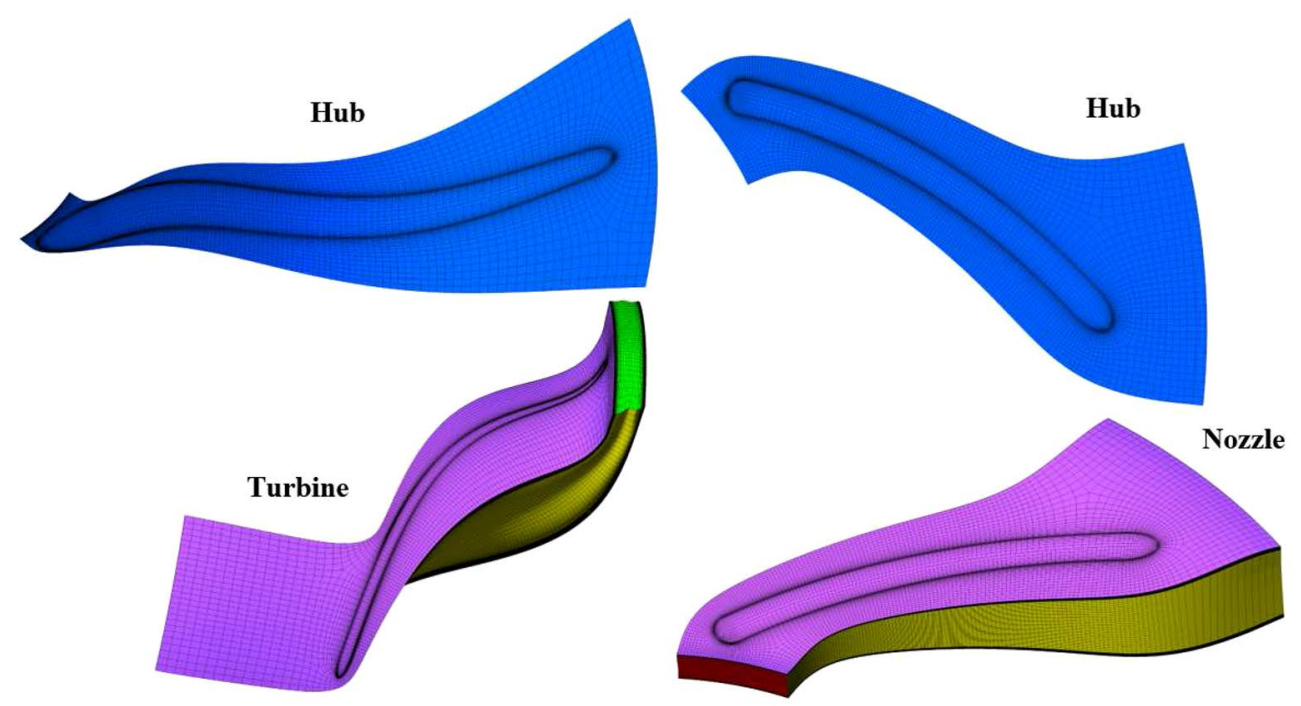

(a)

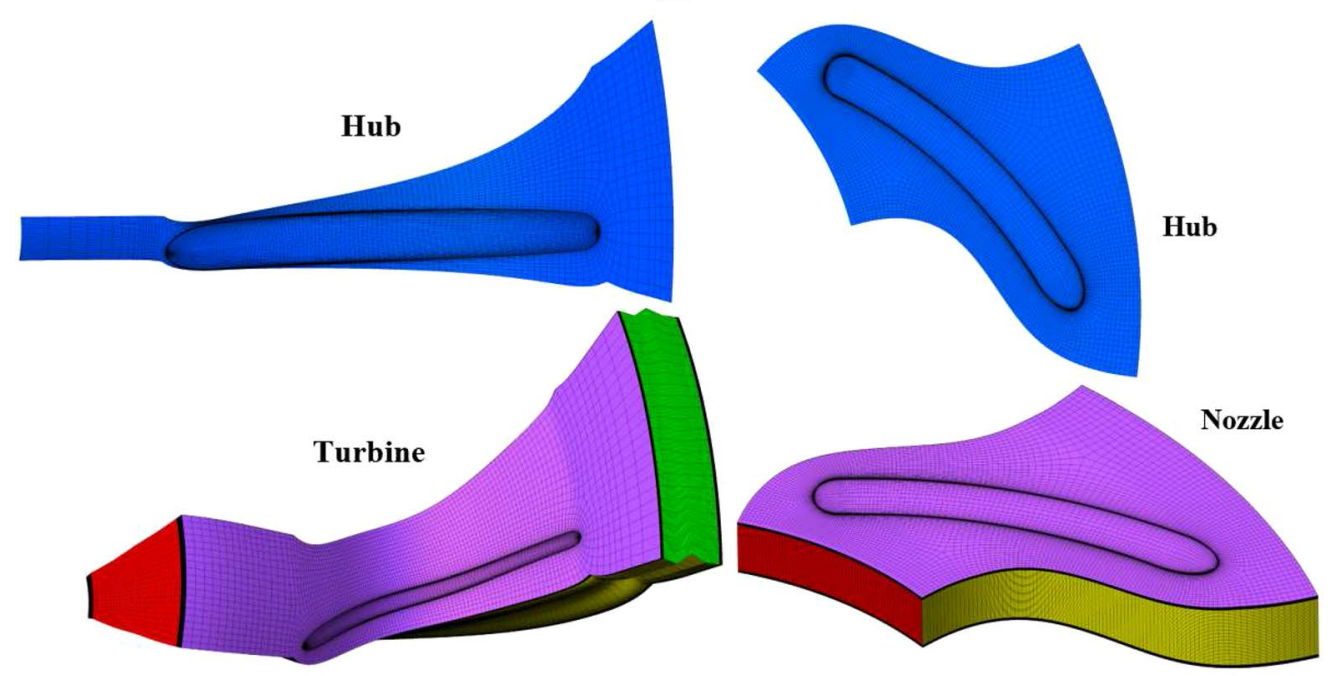

(b)

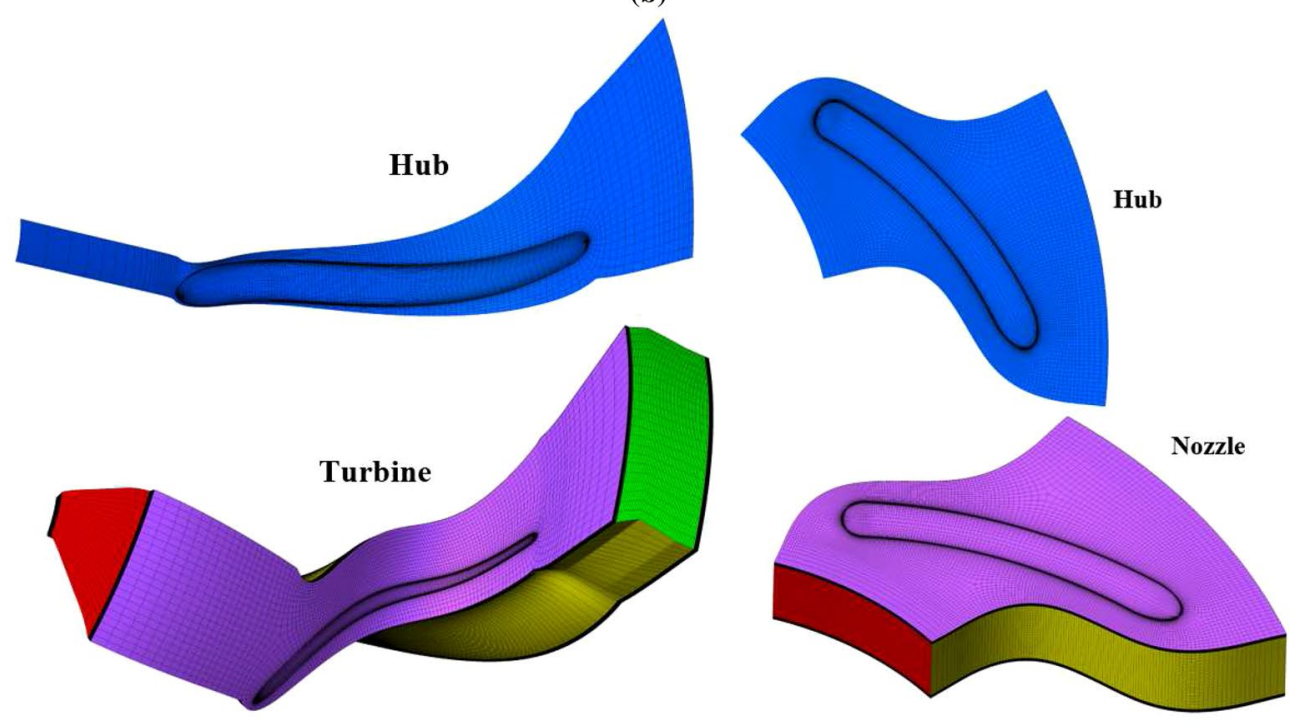

(c)

Fig. 13 The 3D mesh of nozzle and turbine passages a Model 1, b Model 2, c Model 3 


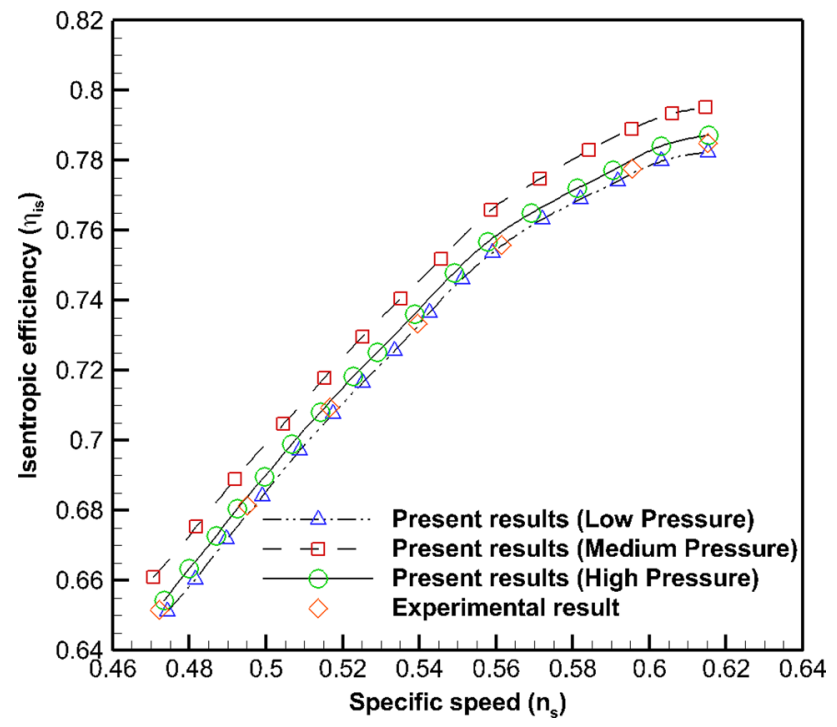

Fig. 14 Comparison of the present numerical results with experimental investigation [34]

the non-dimensional distance from the wall [29], is also considered to solve the boundary layer effect in the vicinity of the turbine blade and flow passages as well. The opted $y+$ value for all the models is mentioned in Table 5.

\subsection{Boundary conditions}

- The total pressure $(16,8$, and 4.5 bar $)$ and total temperature $(80,60$, and $48 \mathrm{~K})$ is set at the nozzle inlet.

- The static pressure is opted at the turbine outlet.

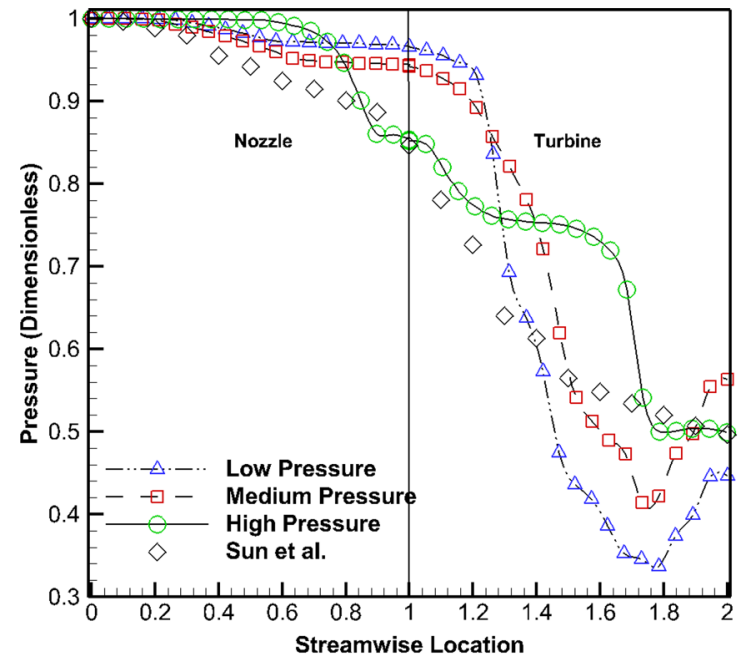

(a)
- The walls (hub, blade, shroud, etc.) are assumed to be adiabatic, hydraulically smooth, and no-slip condition.

- The rotational periodicity is applied along the circumferential direction for nozzle and turbine flow passage to reduce the computational overhead.

Figure 11 illustrates the details of the computational domain. The analysis of the diffuser is not included in this study.

\subsection{Numerical set-up}

The three-dimensional compressible RANS equation with finite volume solver is used for numerical simulations using commercial CFD platform ANSYS CFX. The Navier-Stokes equations are discretized using a secondorder upwind advection scheme. The advection terms are discretized using high-resolution scheme, whereas the transient terms are discretized with second-order backward Euler scheme. Firstly, the interface between the nozzle and turbine is modeled as a stage frame change (mixing plane) with automatic pitch change to compute the rotation of turbine in steady-state condition. In this method, the fluid around the blade is set as a moving reference frame, whereas the blade and hub are assumed to be stationary with respect to the inner fluid. In this way, there is no need for grid movement during steady-state simulation. Secondly, a sliding mesh method has opted during transient blade row simulation with automatic pitch change. The flow through the turbine is analyzed in a rotating coordinate system to visualize the rotating effect. Therefore, the transient

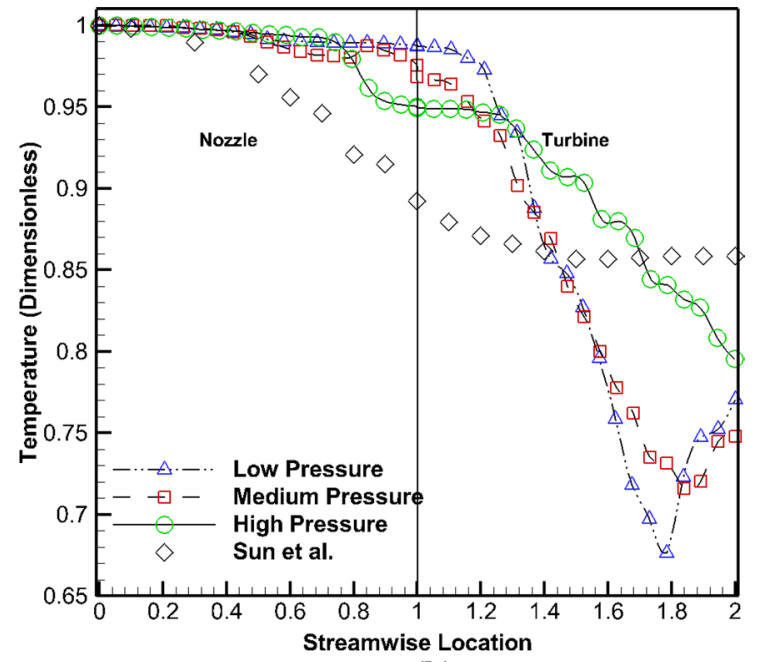

(b)

Fig. 15 Validation of the present results. a Dimensionless pressure, $\mathbf{b}$ dimensionless temperature along the streamwise location [35] 
Fig. 16 Pressure contours at 0.50 span

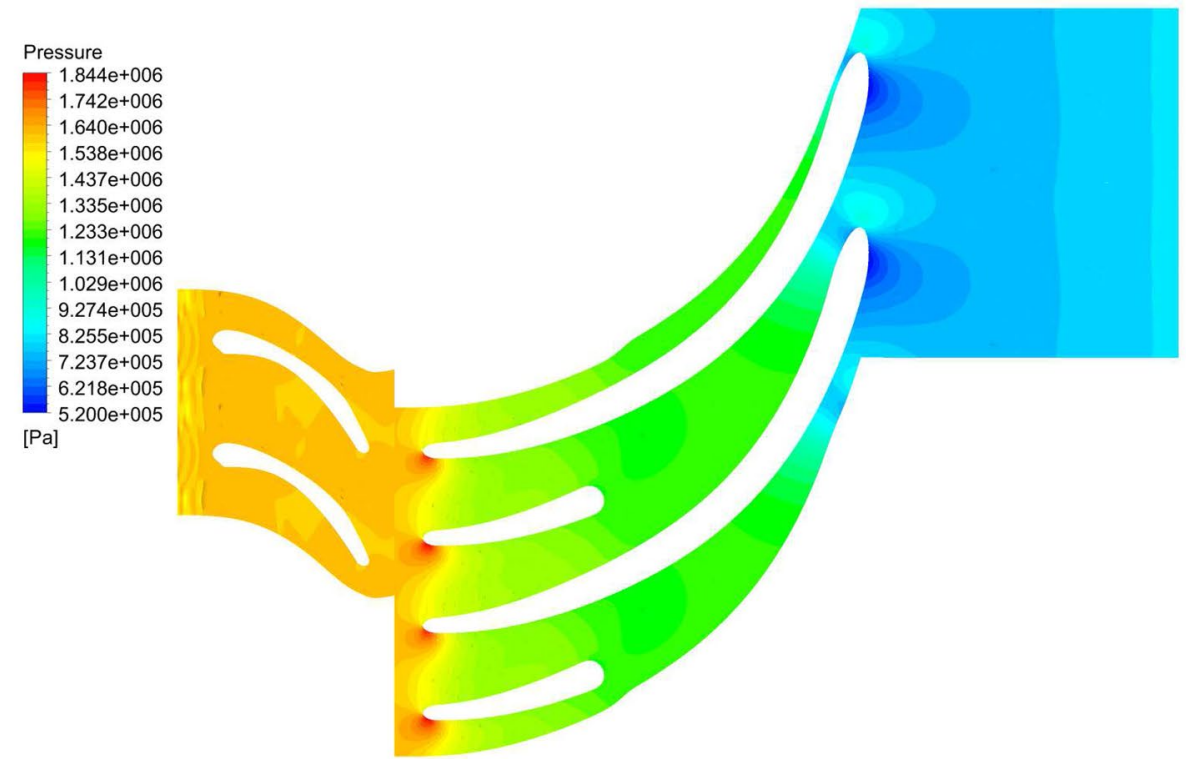

(a) High-pressure

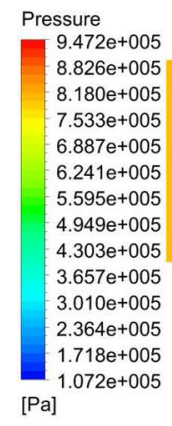

Pressure

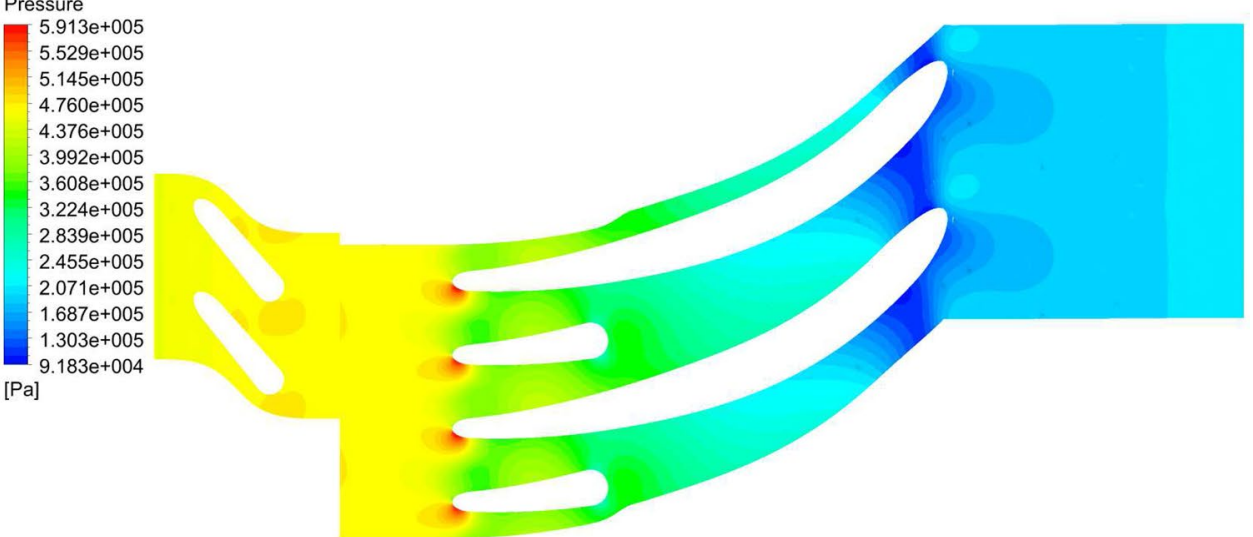

(c) Low-pressure rotor-stator boundary condition is imposed at the interface of the two domains. The rotor domain rotates at each time step which is defined by the pitch ratio. Thus, the boundary nodes of stator and rotor domain slide with respect to each other. Transient simulations are run using the passing period and second-order backward Euler transient scheme. The maximum number of loops is fixed to be 10. This methodology is realistic and more demanding [30]. The turbulence intensity is set to be $5 \%$. The convergence and conservation criteria are set when normalized residuals are less than $10^{-6}$ and $10^{-5}$, respectively. It takes approximately 180 hours to get a converged solution using Intel`Xeon`CPU E5-1660 v3 @ $3.00 \mathrm{GHz}$ with $64 \mathrm{~GB}$ RAM. 


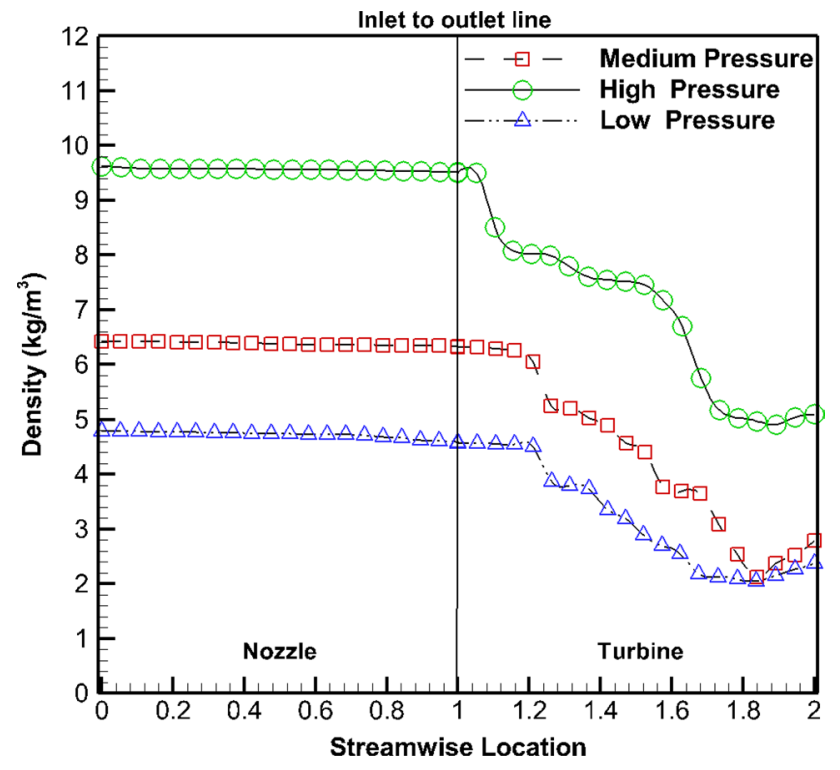

Fig. 17 Area-averaged density variation along the streamwise location

\subsection{Turbulence model and governing equations}

The two-equation eddy viscosity-based $k-\omega$ SST turbulence model has opted to solve the mass, momentum, and energy equations for the transient blade row analysis. The viscous work term is also considered while solving the energy equation. This model is used due to its better capability for automatic wall treatment of the nodes near the wall to capture the turbulence closure and flow separation [31]. The model can obtain the turbulence closure and flow separation effects on the eddy viscosity and accurately predict the turbomachinery flows having boundary layer separations $[32,33]$. It combines the advantages of two commonly used turbulence models: the $k-\omega$ model and $k-\varepsilon$ model. The $k-\omega$ model can predict the flow near-wall region (viscous sub-layer), whereas $k-\varepsilon$ model in wakes and free-shear regions in the outer boundary layer (bulk flow). A blending function confirms the smooth transition between the two models which controls the eddy viscosity by limiting the turbulence shear stress. In this aspect, the $k-\omega$ SST model can predict the boundary layer through the passage (excellent turbulent boundary layer modeling behavior), in which $\omega$ near the wall is more stable than that of the $\varepsilon$. The discretization schemes of different governing equations are mentioned in Table 6.

\subsection{Modeling based on real fluid properties}

The fluid properties at high pressure and the ultra-low temperature never follow the ideal gas laws. The implementation of the equation of state significantly affects the accuracy of the numerical solution. In this regard, the Peng-Robinson real gas equation of state is opted [33].

\subsection{Grid independence test}

To verify the accuracy of the numerical results, a grid independence test is carried out. Figure 12 represents the isentropic efficiency variation for four different grid resolutions. It is observed that the change in isentropic efficiency is nearly unchanged for third and fourth grid size. After that, the computational time is increased with less effect on the isentropic efficiency. Therefore, as a compromise between the accuracy of the results and computational time, the third grid size is opted for the remainder of the analysis as shown in Fig. 13.

\subsection{Model validation}

The validation of the computational results is necessary for the reliability of the solution. There are very few experimental works reported in the open literature for helium turboexpander operating at cryogenic temperature. The present results are validated with experimental results of high-pressure helium turboexpander with Chakravarty et al. [34] at a different rotational speed. Figure 14 shows that the present numerical results agree well with experimental results. Additionally, the results are also validated with numerical results of Sun et al. [35]. Figure 15 illustrates the comparison of the dimensionless pressure and temperature variation in the turboexpander along the streamwise location. It indicates that the dimensionless pressure variation has a good agreement with Sun et al. [35]. However, there is deviation in dimensionless temperature plot which occurs because of different fluid and operating conditions. Conclusively, the present numerical results are reliable.

\section{Results and discussion}

Based on the aforementioned numerical methodology and assumptions, this section illustrates the numerical results to visualize the fluid flow and thermal behavior at different inlet pressure and temperature inside the turboexpanders. It is reasonable because of random change in molecular viscosity and density of the 
Fig. 18 Mach number variation at 0.5 span

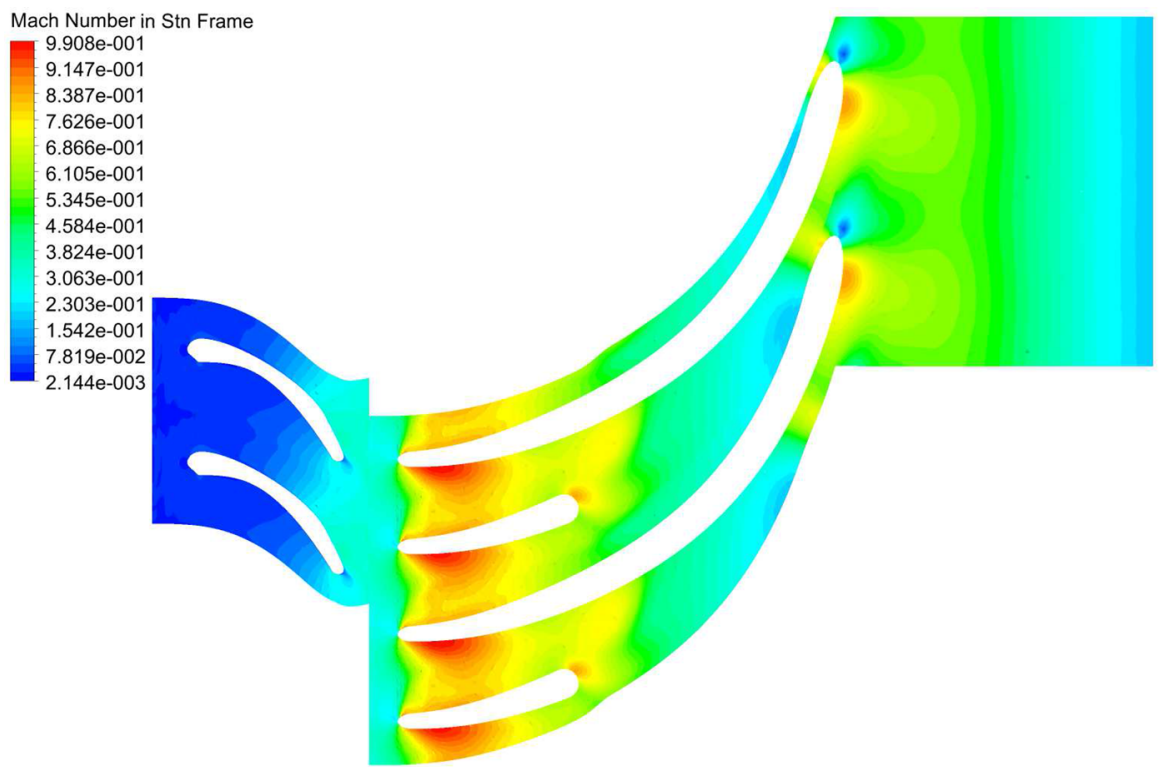

(a) High-pressure

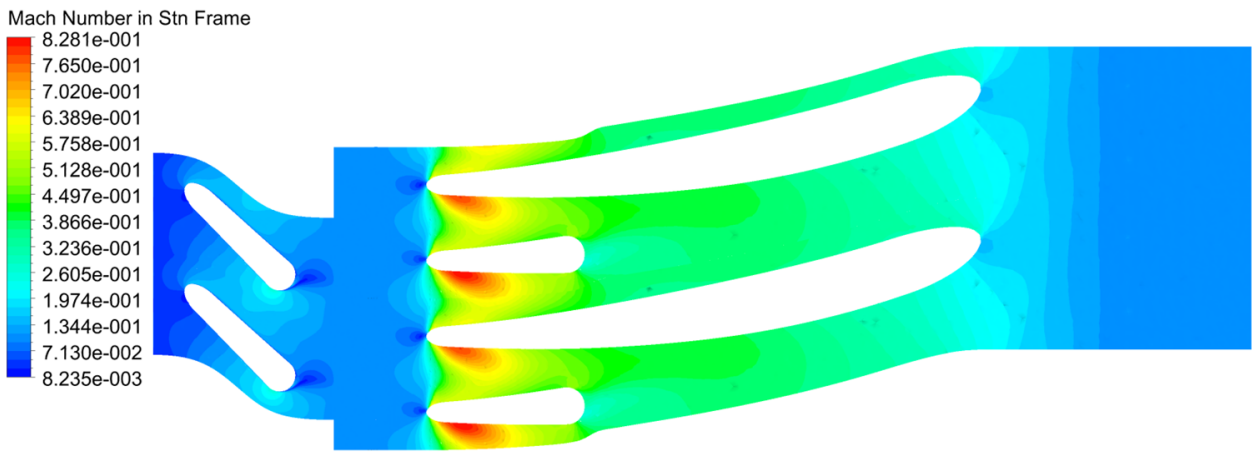

(b) Medium pressure

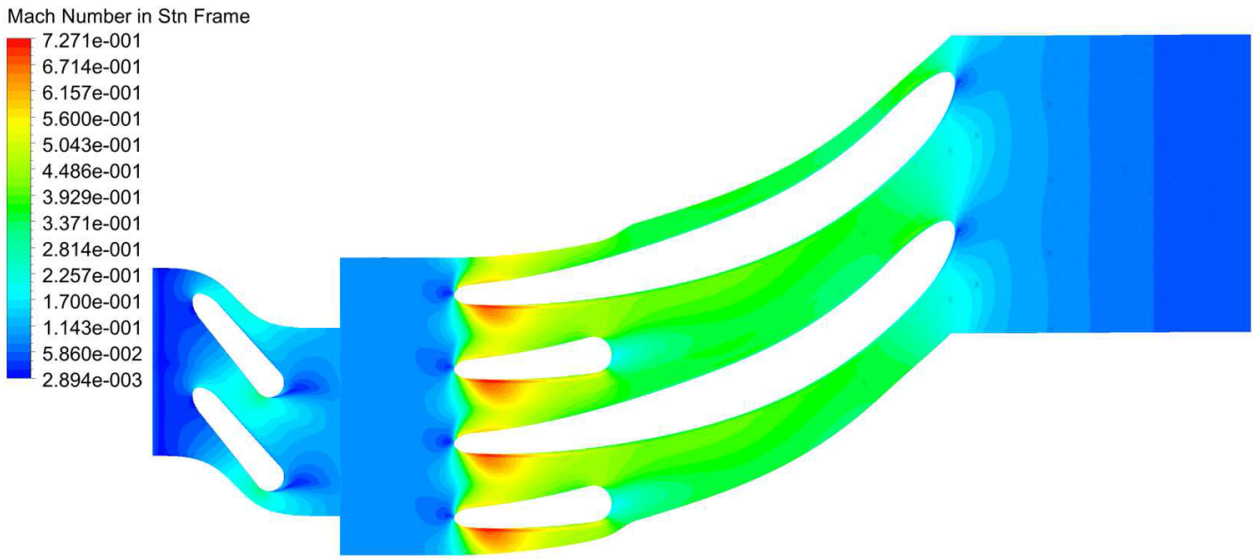

(c) Low-pressure superfluid helium at cryogenic temperature. The study is also focused on to differentiate the effect of splitter blades on the performance of the turboexpander. The results are illustrated for pressure variation, Mach number, Reynolds number, velocity vectors, etc. Additionally, the attention is extended to study the thermal behavior of the fluid such as temperature, Prandtl number, density, static enthalpy, and static entropy. 


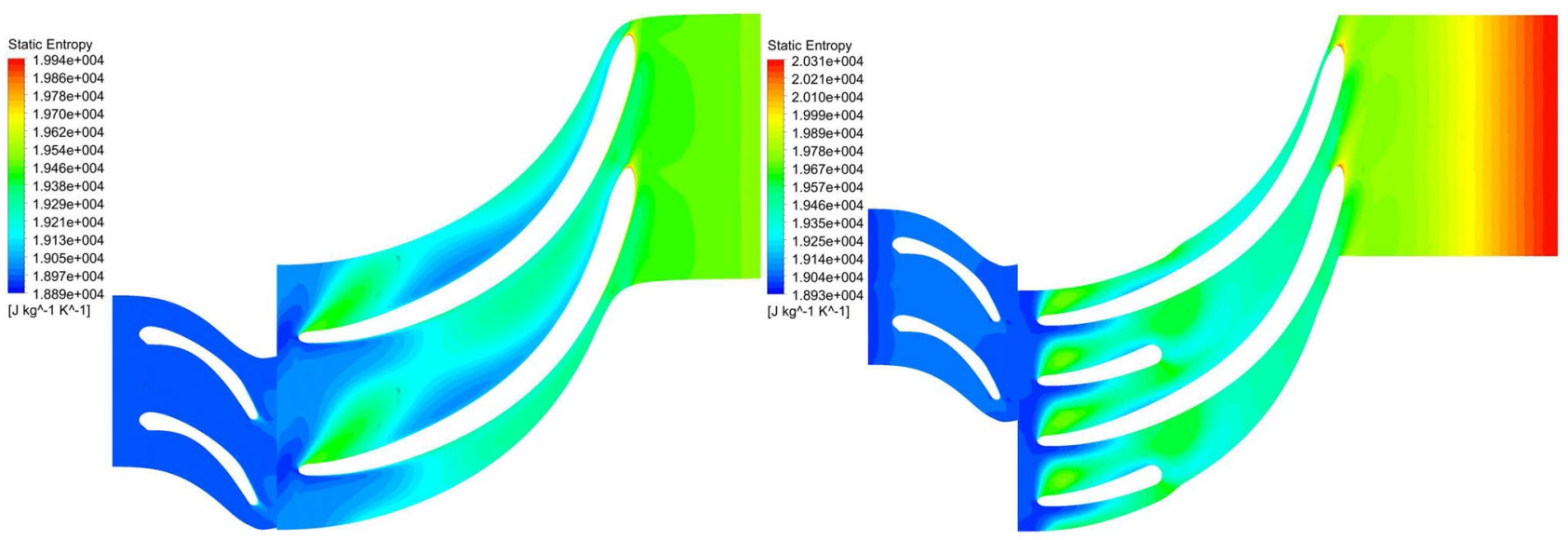

(a) Case 1

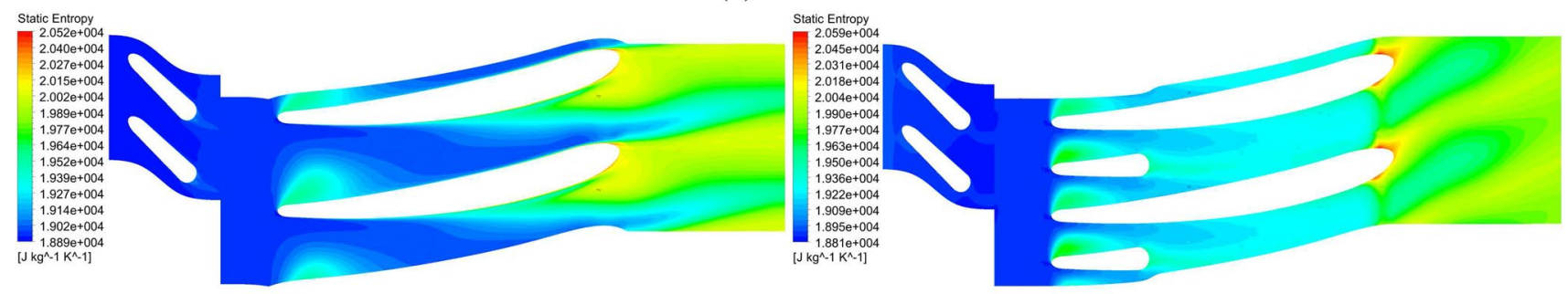

(b) Case 2

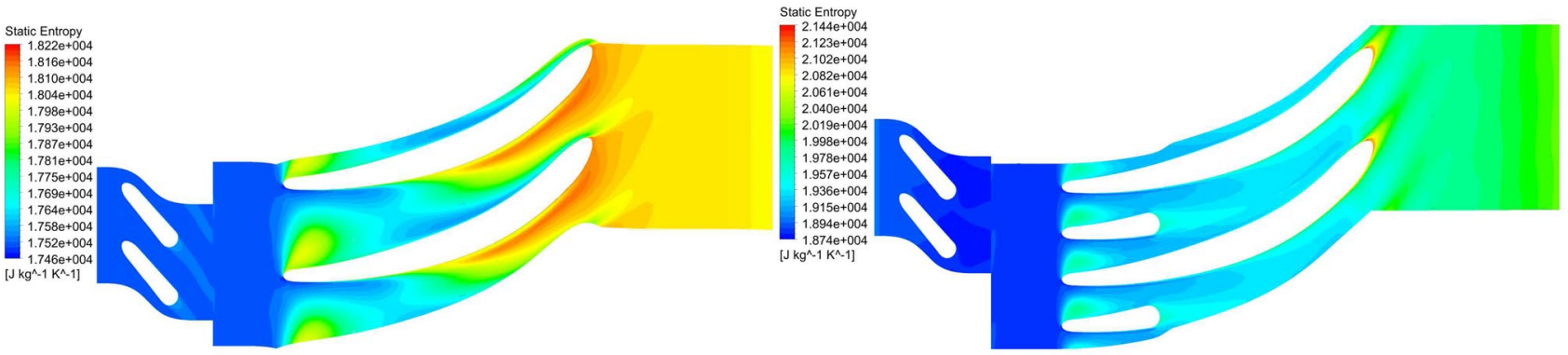

(c) Case 3

Fig. 19 Static entropy variation at 0.5 span

\subsection{Effect of splitter blades on the fluid flow properties}

The pressure drop inside the turboexpander depends on the design of the blade profile of the nozzle and turbine. The flow must be in the subsonic regime throughout the domain. Therefore, the pressure ratio is the limiting factor in defining the flow condition inside the turboexpander. Figure 16 represents the pressure distribution at $50 \%$ blade height (mid-span) for three different inlet pressures. It shows the uniform pressure distribution except at the trailing edge where a local pressure drop exists. At this place, the higher temperature drop takes place which is further increased due to the increase in pressure energy after the trailing edge (Fig. 24). The pressure drop inside the high-, medium-, and low-pressure turboexpander is 8.00 bar, 3.50 bar, and 2.50 bar, respectively. As a result, the energy transformation takes place which reduces the static enthalpy of the fluid and produces the cooling effect. The approximately uniform pressure distribution states that the preliminary design of the turboexpander is adequate for all the three models. Since the pressure of the fluid changes, it must affect the quantitative variation of density (Fig. 17). It is noticed that the density variation is higher for high-pressure fluid as compared to medium- and low-pressure fluid. Figure 18 represents the Mach number contours in the flow passage at 0.50 span for a fixed pressure ratio. Since 
Fig. 20 Velocity vectors in the high-pressure turbine with and without splitter blades

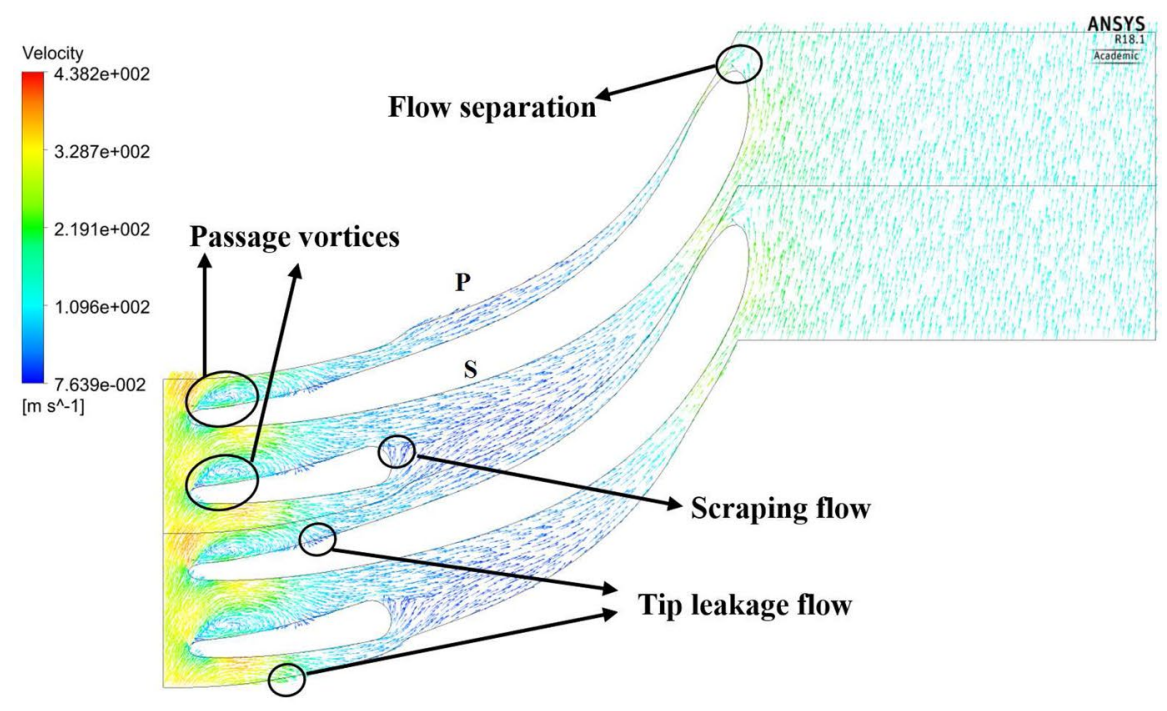

(a) Span 0.10

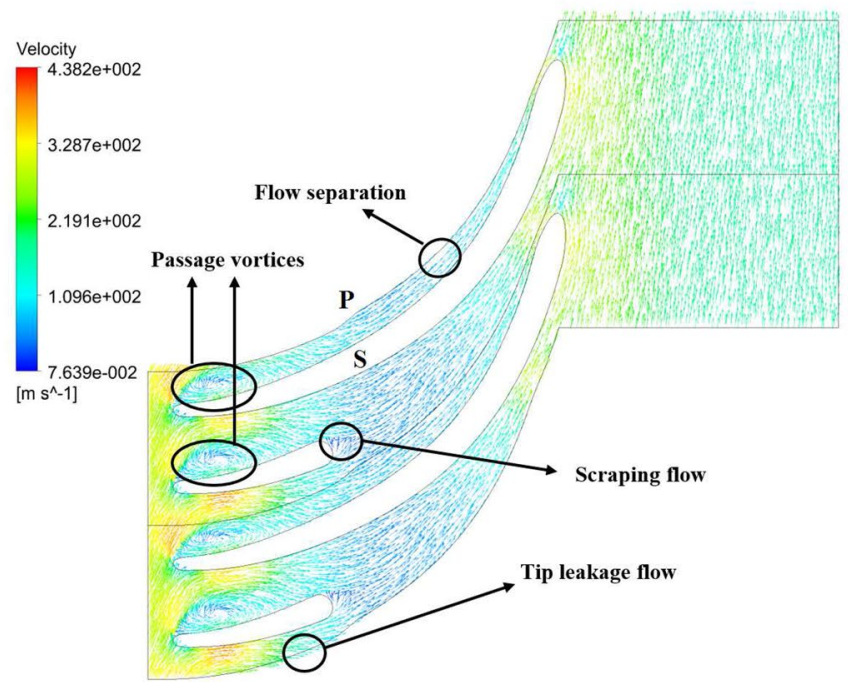

(b) Span 0.50

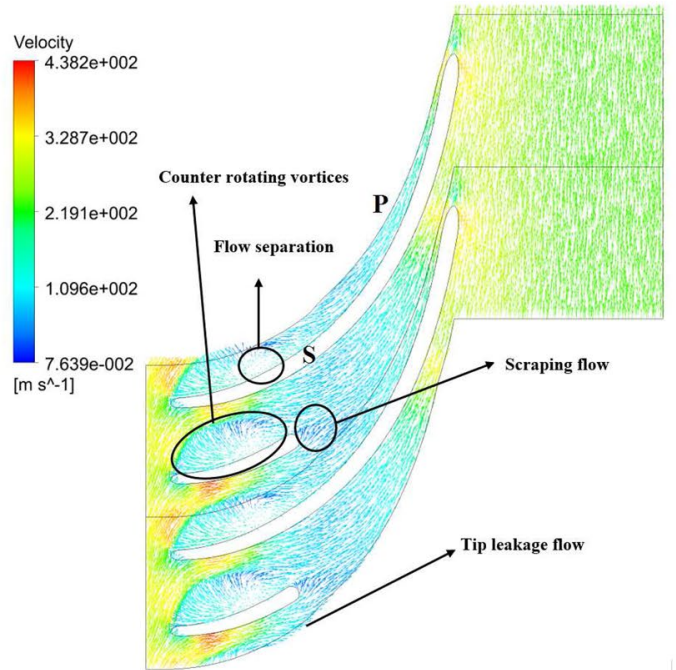

(c) Span 0.90 
Fig. 21 Velocity vectors in the medium pressure turbine with and without splitter blades

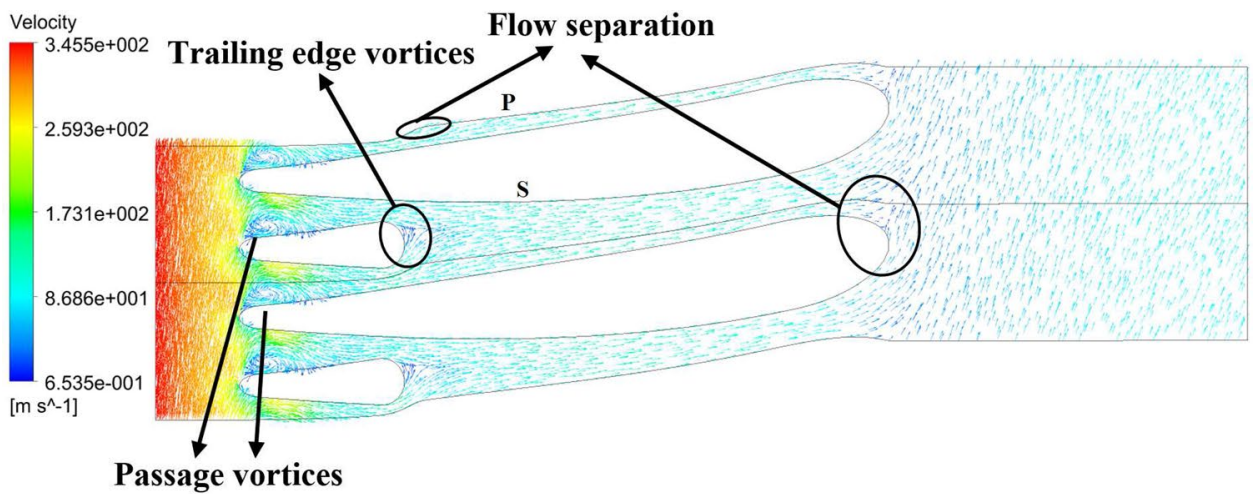

(a) Span 0.10

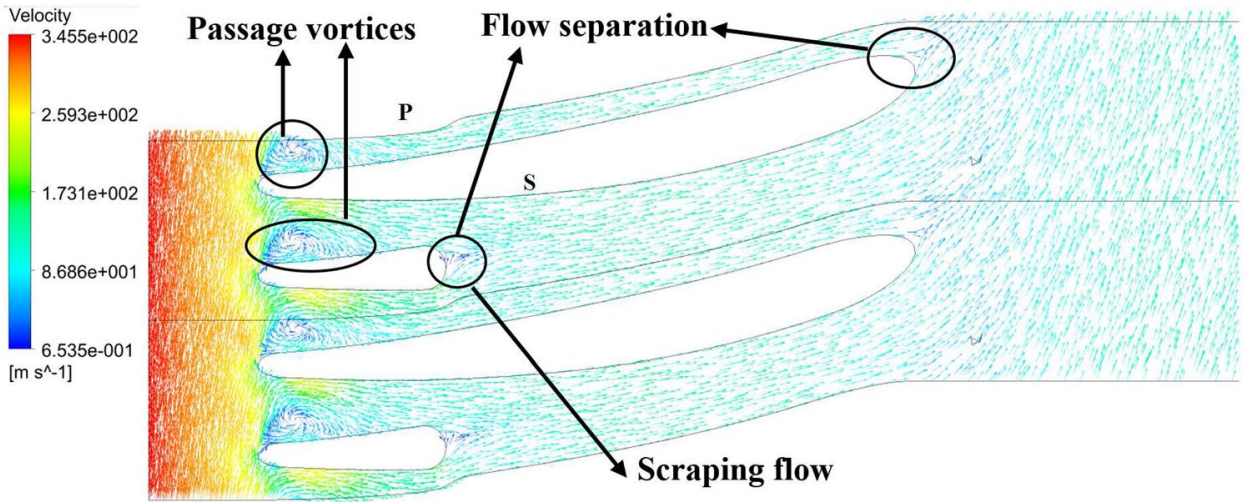

(b) Span 0.50

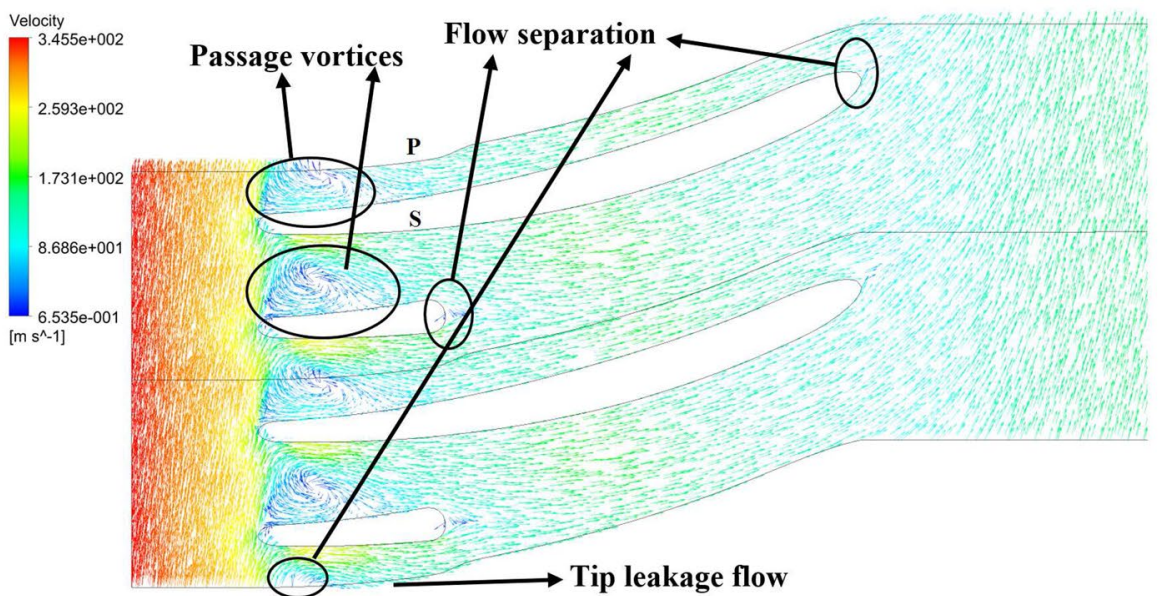

(c) Span 0.90 the turboexpanders are designed to operate in the subsonic regime, the contours justified the validity of the design procedure. The Mach number at the inlet of the turbine is $0.81,0.74$, and 0.63 for high-, medium-, and low-pressure turboexpanders, respectively.

The boundary layer formation, passage vortices, freestream layer, etc., are responsible for the increase in entropy.
Figure 19 represents the static entropy contours at 0.50 span with and without splitter blades. Since the viscous friction increases due to the generation of vortices in the flow passage which also increase the entropy of the fluid. It is reduced by placing the splitter blades. It is also observed that the mixing of fluid streams from pressure and the suction side of the blade increases the shear strain which leads 
Fig. 22 Velocity vectors in the low-pressure turbine with and without splitter blades

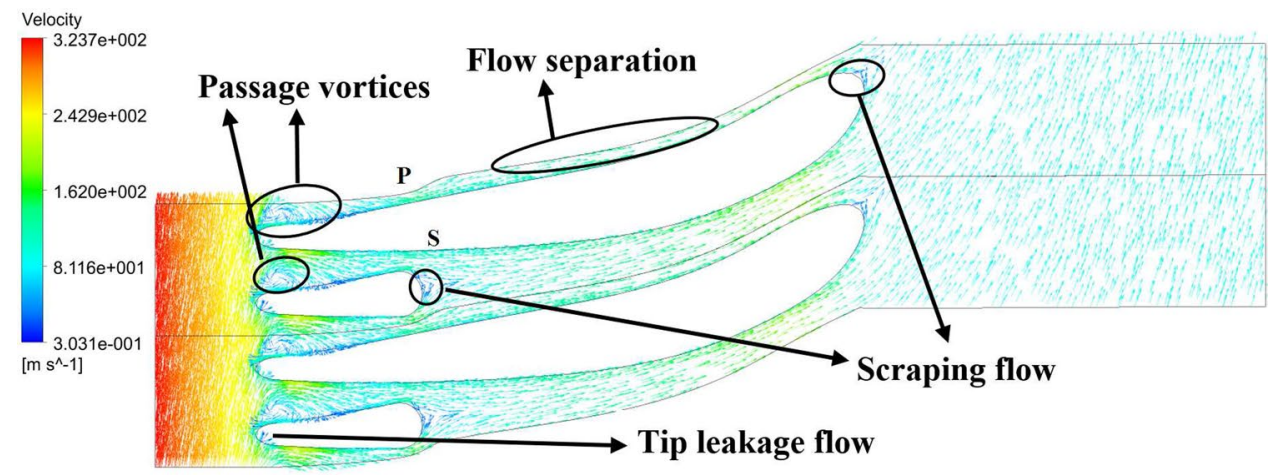

(a) $\operatorname{Span} 0.10$

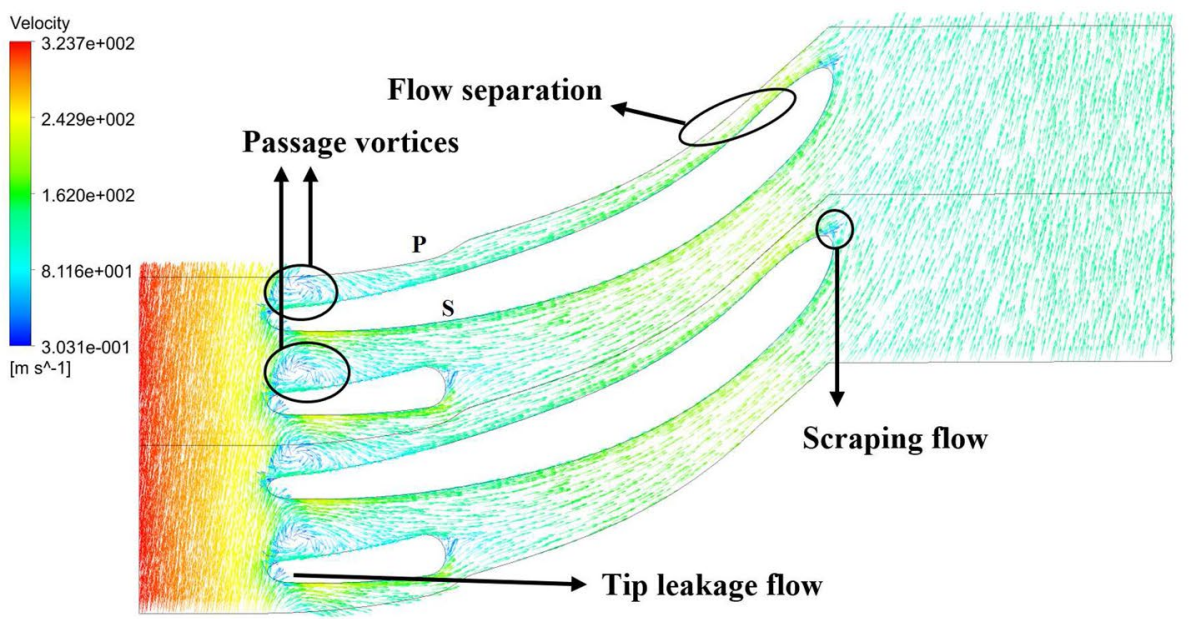

(b) Span 0.50

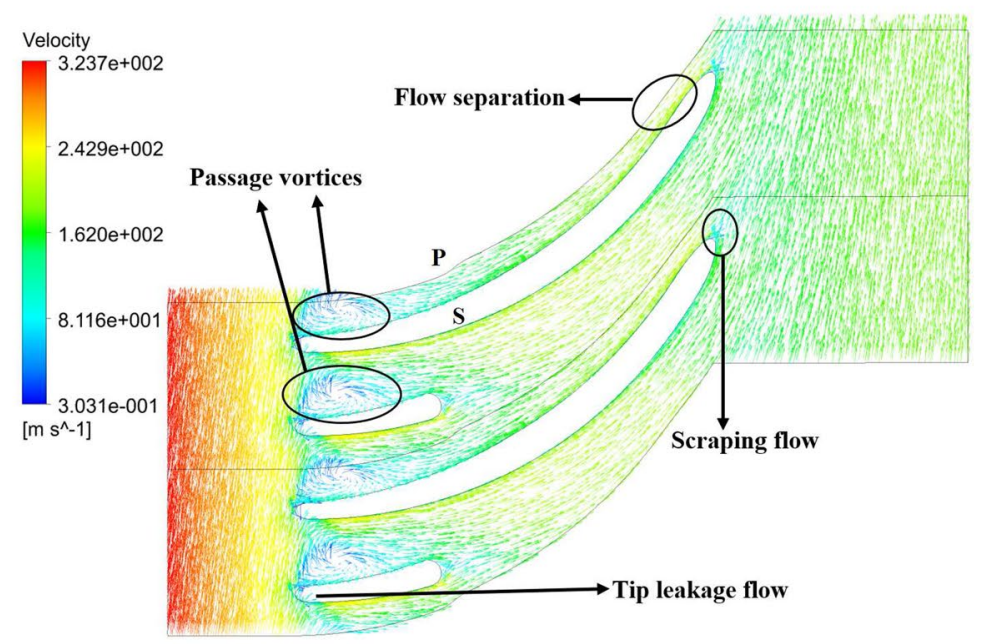

(c) Span 0.90

to increase the entropy. The entropy increases for the portion of the blade where the tip leakage flow, flow separation, and vortices are generated.

Figures 20, 21, and 22 represent the velocity vectors inside the turbines with splitter blades at different blade heights. It depicts the flow separation, vortex generation, scraping flow, passage vortices, tip leakage flow, etc., at different locations. For the high-pressure turbine, it is noticed that the flow separation takes place near the trailing edge for 0.10 span (Fig. 20a). At higher spans, the 


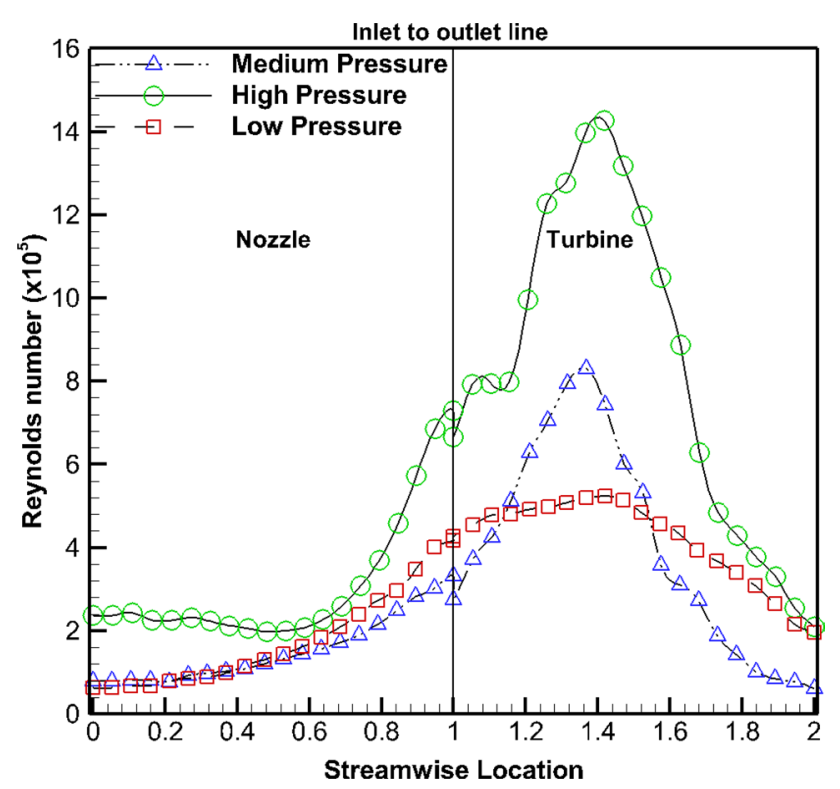

Fig. 23 Variation of Reynolds number along the streamwise location

flow separation occurs near the leading edge. The intensity of passage and counter-rotating vortices is reduced near the splitter blades. However, the scraping flow occurs near the trailing edge of the splitter blades which accounts less amount of losses as compared to passage and counterrotating vortices occur in the mainstream without placing the splitter. The tip leakage flow is also appeared near the blade tip clearance due to the pressure difference between the pressure and the suction side.

For medium pressure fluid, the flow separation occurs at the pressure side and trailing edge of the main blade. At higher spans, the intensity of passage vortices increases, whereas the trailing edge vortices diminishes near the trailing edge of the splitter blades. For low-pressure fluid, the passage vortices appear near the leading edge. The flow separation and the intensity of vortices are relatively increased at higher spans. Additionally, the secondary flow occurs in the vicinity of the blade wall boundary layer due to the relatively higher radial component of Coriolis acceleration as compared to the radial pressure gradient. The similar phenomenon is also observed by Zangeneh et al. [36] for the centrifugal impeller. The secondary flow also appears due to the induced pressure gradient by the curvature of the blade profile. The tip leakage flow introduces the non-uniformity and turbulence in the fluid flow and a major cause of losses in the rotating machines. Conclusively, it can say that the addition of splitter blades is helpful for better flow stability and comparatively less passage vortices near the leading edge. However, some amount of flow separation and scraping flow appears near the trailing edge of the splitter blade.

Reynolds number is a dimensionless parameter defined to identify the fluid flow condition. It is defined as:

$\operatorname{Re}=\frac{\rho v D_{\mathrm{h}}}{\mu}$

Figure 23 illustrates the variation of the area-averaged Reynolds number along with the streamwise location. The change significantly depends on the pressure drop, hydraulic diameter, and kinematic viscosity of the fluid. Since the low-temperature fluid approaches toward the boiling point temperature, the density is relatively higher which reduces the kinematic viscosity of the fluid. Reynolds number is maximum for high-pressure fluid near the turbine inlet. For medium- and low-pressure fluid, the variation is approximately similar inside the nozzle. However, it is higher for medium pressure fluid inside the turbine.

\subsection{Variation of thermal properties}

Figure 24 represents the comparison of variation of temperature contours of the high-, medium-, and low-pressure fluids at $0.50 \mathrm{span}$. The local temperature drop near the leading edge takes place due to the placement of the splitter blade, which reduces sharply in the main flow by placing the splitter blades due to relatively higher enthalpy drop of the fluid. Since the decrease in temperature at cryogenic temperature captivates different properties governed by the corresponding enthalpy drop (Fig. 25a), the refrigerating capacity of the turboexpander defines the efficiency of the system. Although the phenomenon depends on the pressure drop and the state of the fluid at corresponding pressure and temperature, the temperature drop decreases when the working fluid advances toward its boiling point. In those cases, the thermodynamic properties of the cryogenic fluids are randomly changed which severely affect the fluid flow and thermal behavior during the expansion process. The high-pressure helium has higher enthalpy as compared to the other cases. Therefore, the enthalpy drop is maximum for this case which is the primary reason for more substantial temperature drop inside the turboexpander. Since the working fluid gains the pressure energy after passing the trailing edge, it causes a local temperature rise in this zone (Fig. 26).

The adiabatic or throttling effect hypothesis governs the expansion of the fluid inside the turboexpander. The law of conservation of energy states that during the expansion process, the enthalpy of the fluid decreases. For the present case, the expansion process is illustrated 


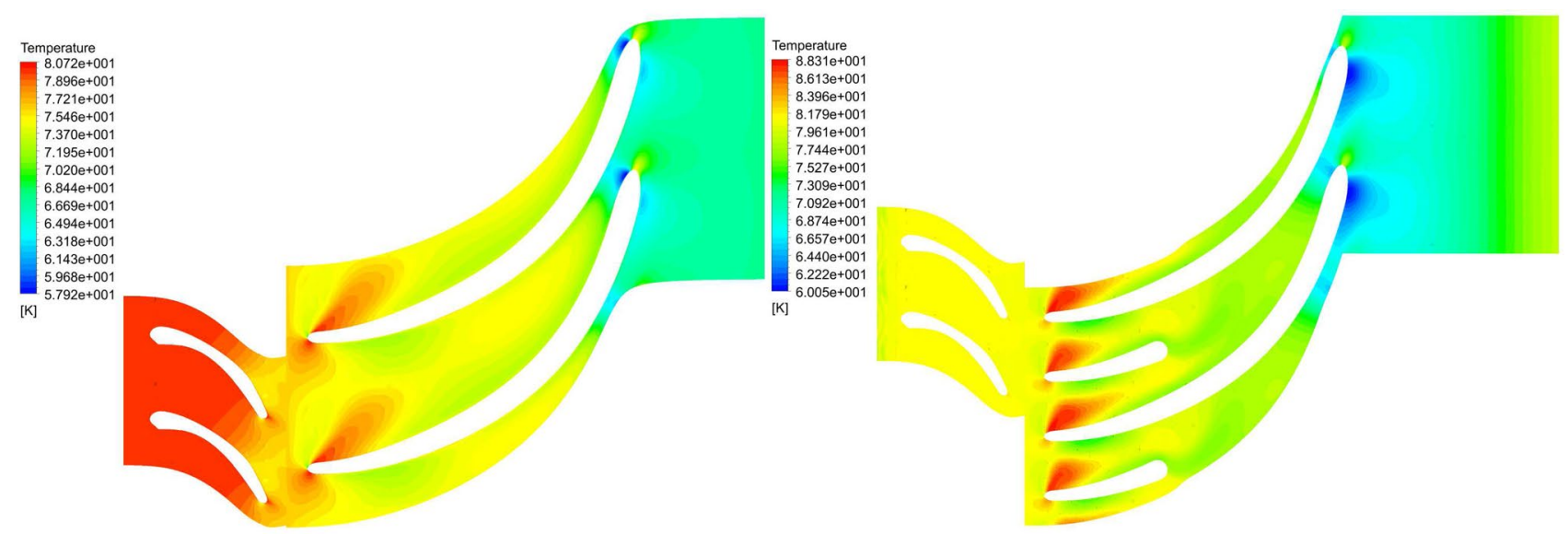

(a) High-pressure

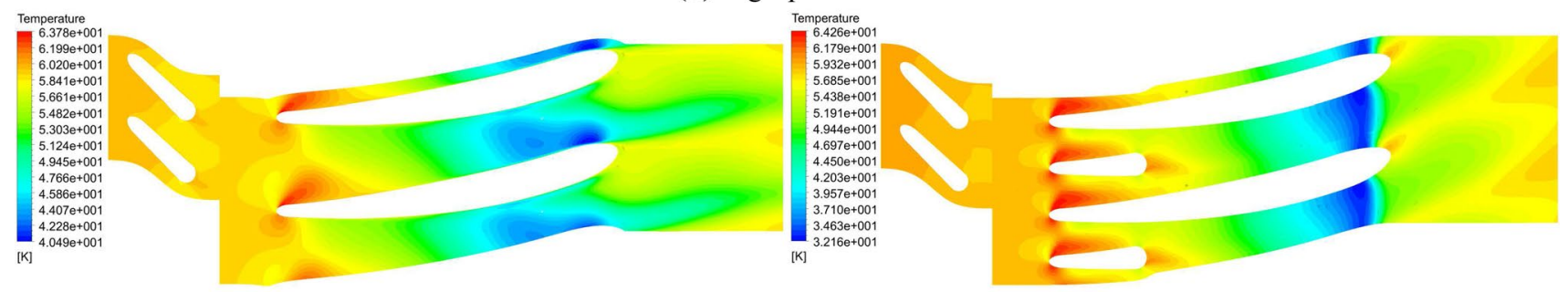

(b) Medium pressure

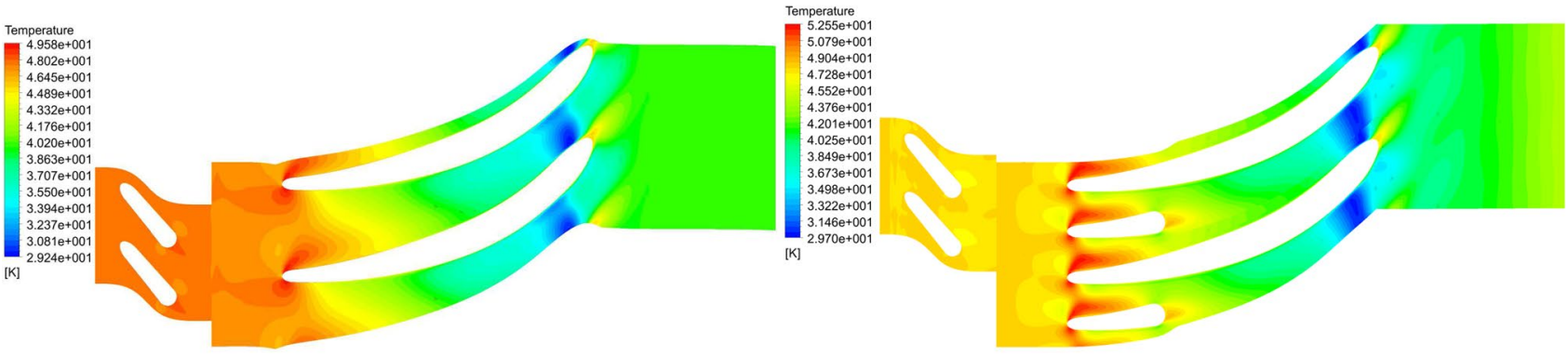

(c) Low-pressure

Fig. 24 Temperature contour distribution at 0.50 span

through the pressure-static enthalpy and temperaturestatic entropy variation (Fig. 27). The actual expansion process is always different due to the irreversibility of the system. It also increases the entropy of the system (Fig. 19). The ideal properties of the fluid are obtained using REFPROP` which are based on the corresponding pressure and temperature of the fluid along the streamwise location. In this regard, the isentropic efficiency $\left(\eta_{\text {is }}\right)$ is defined to consider the irreversibility of the expansion process.

The isentropic efficiency is calculated as:

$\eta_{\text {is }}=\frac{h_{\text {in }}-h_{\text {ex }}}{h_{\text {in }}-h_{\text {ex }, \text { is }}}$ where $h_{\text {ex,is }}$ is obtained from outlet pressure and temperature of the corresponding fluid. The isentropic efficiency and power output of the system for a different inlet temperature of the fluids are mentioned in Table 7.

Prandtl number is a dimensionless parameter which is introduced to characterize the thermal condition of the fluid. It is defined as follows:

$\operatorname{Pr}=\frac{\mu C_{\mathrm{p}}}{k_{0}}$

Figure 28 illustrates the Prandtl number variation inside the turboexpander. 


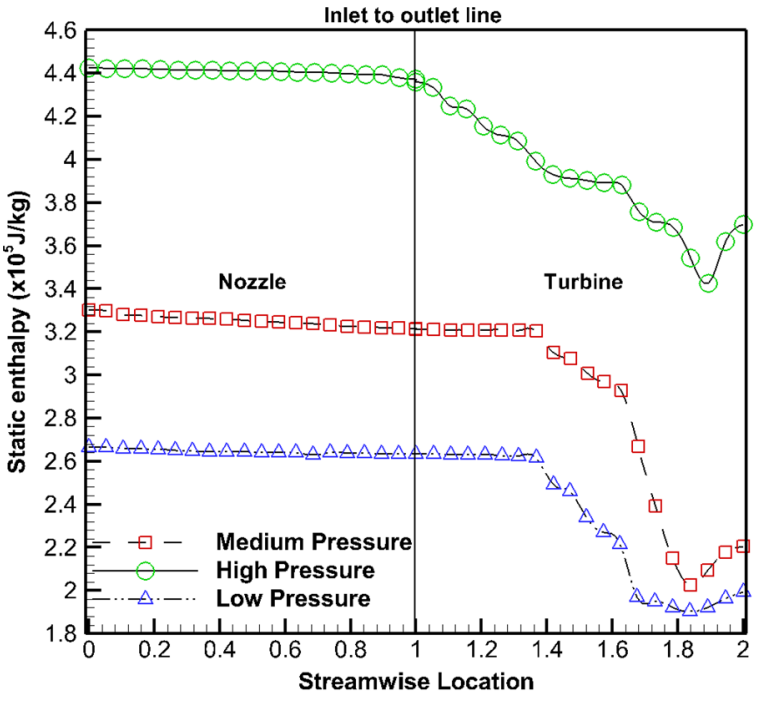

(a)

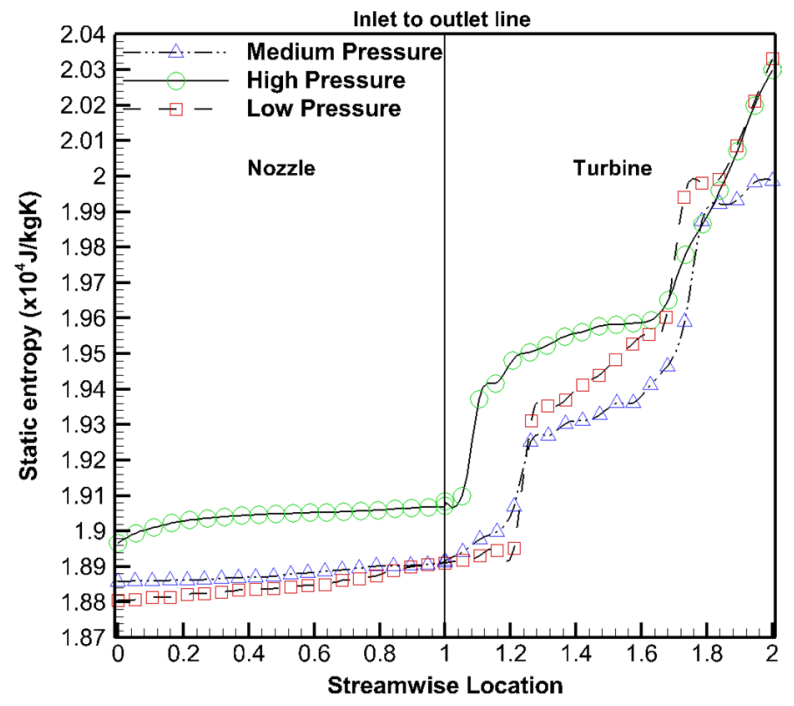

(b)

Fig. 25 Area-averaged a static enthalpy, b static entropy variation along the streamwise location

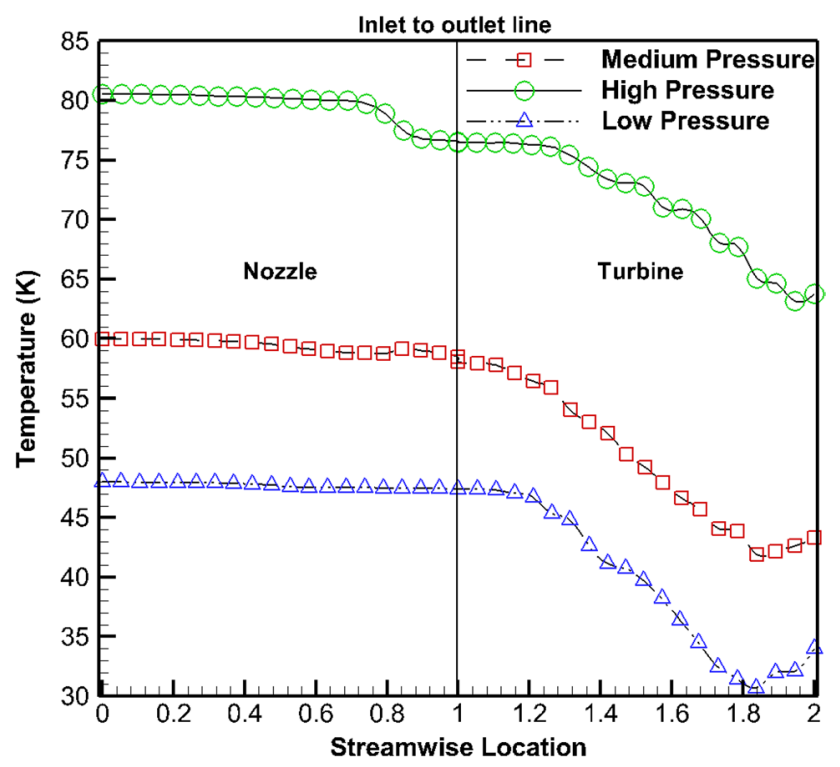

Fig. 26 Area-averaged temperature variation along the streamwise location

\subsection{Effect on TKE and eddy viscosity}

The turbulence intensity is quantifying in terms of TKE in turbulent flow. It can be utilized to characterize the flow effect of eddy viscosity during the fluid flow. It is not defined as a fluid property yet rather remarkably affects the flow behavior and depends on the shape of the domain and free-stream turbulence intensity [33]. Since the SST model overrates the turbulence in the fluid flow which shows the magnitude of eddy diffusion and their distribution. Figure 29 illustrates the variation of TKE along the streamwise direction. It is noted that the TKE is in the lower side in the nozzle for all the cases. It happens due to the stationary component and intensity of TKE is almost identical for medium- and low-pressure fluid. For highpressure fluid, it increases after 0.80 streamwise location. It also depicts the high-pressure fluid as highest TKE near the rotor-stator interface. It is obvious due to relatively high flow turbulence, and passage vortices are appeared in this zone because of the rotational effect of the turbine. The medium pressure fluid shows highest TKE at the trailing edge which is due to the profile of the rotor blade. It can be reduced by placing the diffuser.

Figure 30 illustrates the variation of eddy viscosity at 0.50 span. It is noticed that the intensity of eddy viscosity is highest near the leading edge of the rotor for high and low-pressure fluid, whereas it is higher near the trailing edge for medium pressure fluid. Generally, the intensity increases toward the trailing edge which is advantageous to decrease the losses incurred inside the turbine, but its appearance also depends on the domain structure. 


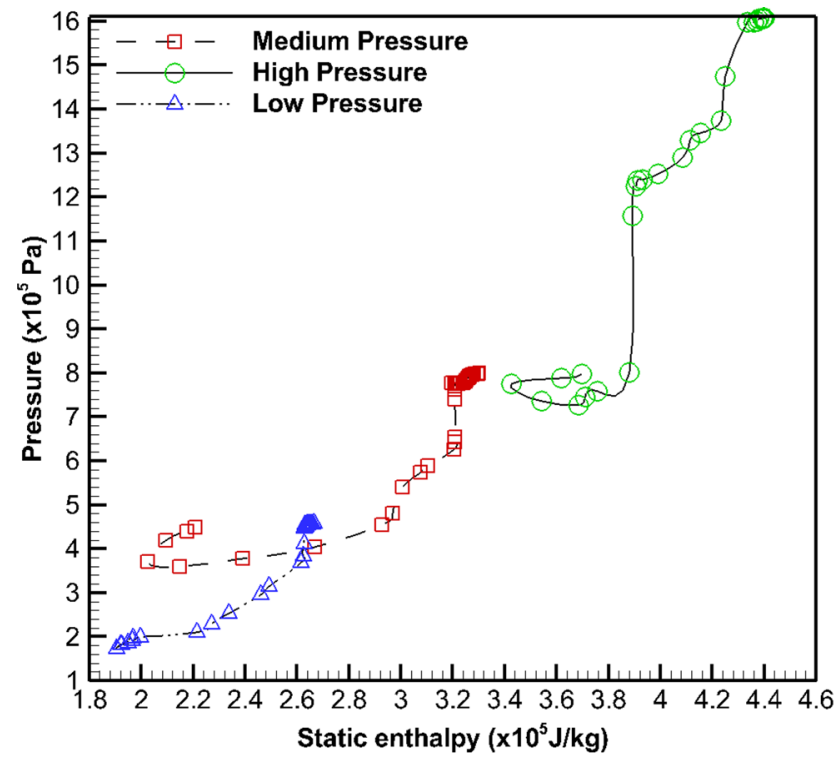

(a)

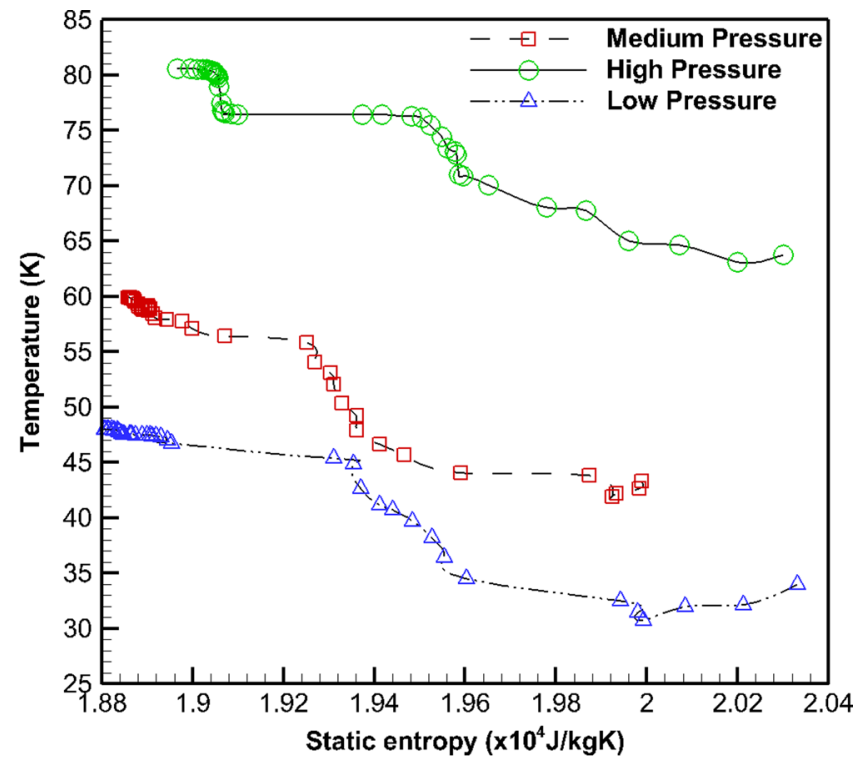

(b)

Fig. 27 a Pressure versus static enthalpy, b temperature versus static entropy variation

Table 7 Performance of the turboexpander with and without the splitter blades

\begin{tabular}{lll}
\hline Fluids & $\begin{array}{l}\text { Isentropic efficiency }\left(\eta_{\text {is }}\right) \\
\text { (blade/splitter) }\end{array}$ & $\begin{array}{l}\text { Power }(\mathrm{kW}) \\
\text { (blade/split- } \\
\text { ter) }\end{array}$ \\
\hline High pressure & $0.748 / 0.751$ & $7.87 / 7.92$ \\
Medium pressure & $0.785 / 0.792$ & $5.24 / 5.32$ \\
Low pressure & $0.768 / 0.773$ & $6.51 / 6.53$ \\
\hline
\end{tabular}

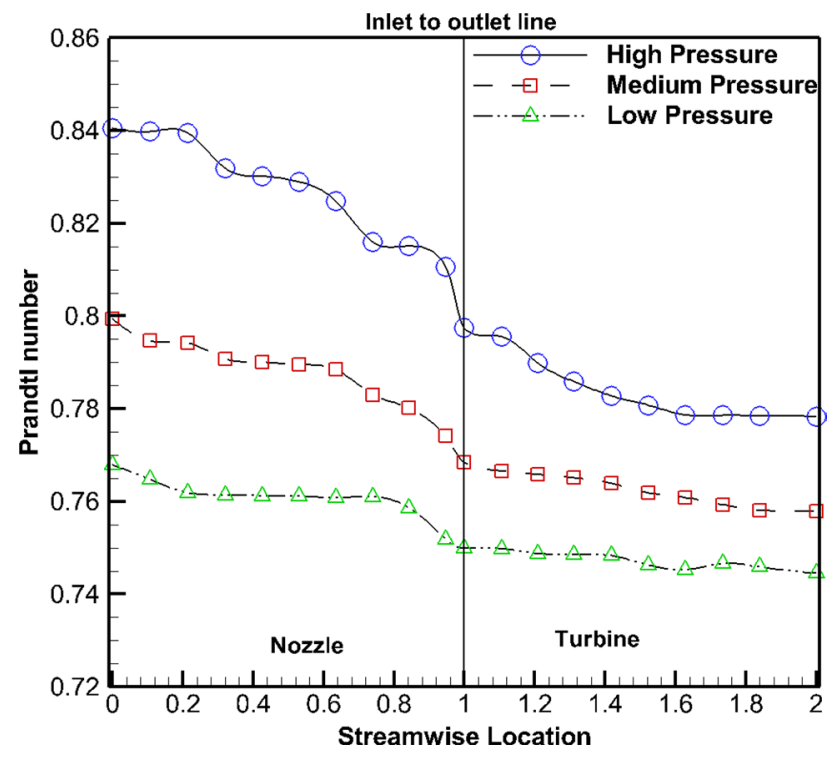

Fig. 28 Variation of Prandtl number along with the streamwise location

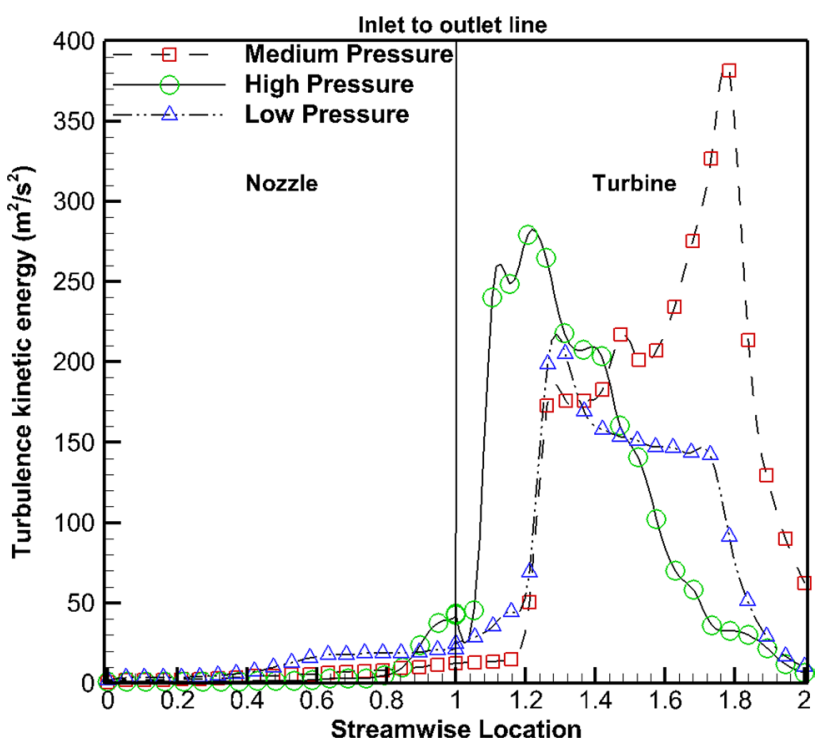

Fig. 29 Area-averaged TKE variation along the streamwise location

\section{Conclusions}

The mean-line design methodology and performance prediction of a radial inflow turbine are explained in this paper. The design methodology is used for the practical design of three turboexpander system used for liquefaction of helium. From the non-dimensional design variables datasets; an ANN and ANFIS model are developed to predict the optimal range in which the turbine has maximum 


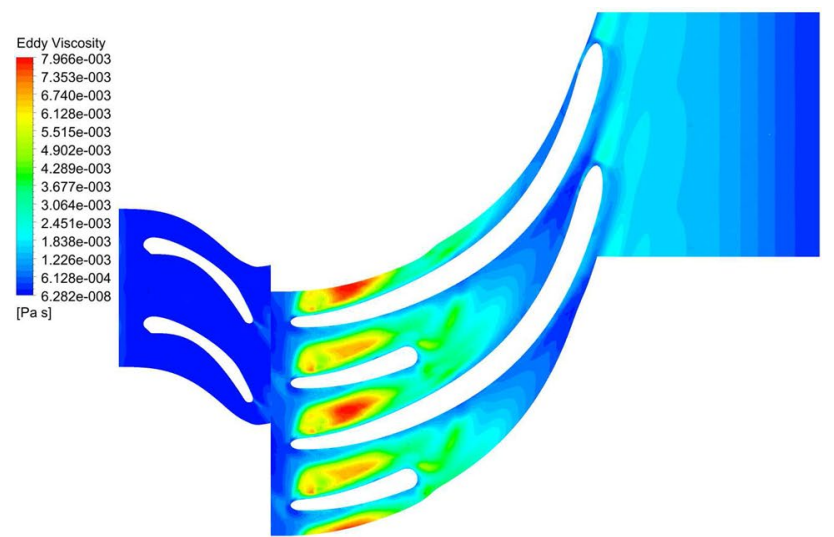

(a) High-pressure

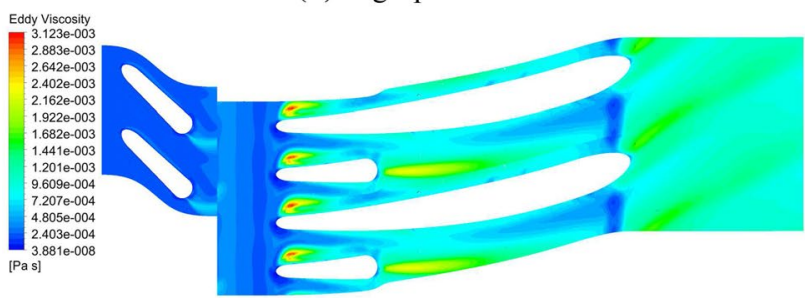

(b) Medium pressure

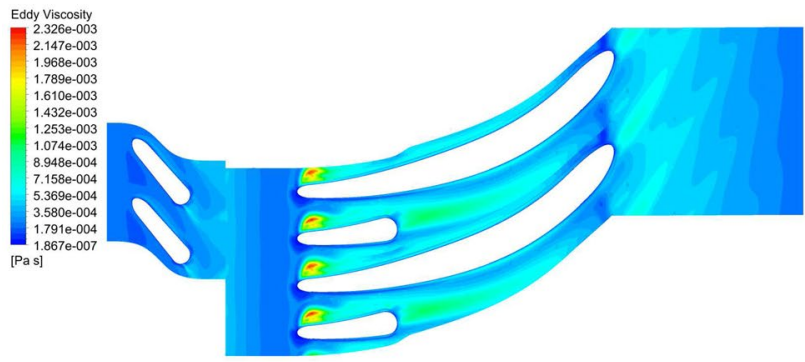

(c) Low-pressure

Fig. 30 Eddy viscosity contours at 0.5 span

efficiency and power output with minimum losses. After that, a comparative study to investigate the effect of splitter blade on fluid flow and thermal characteristics for high-, medium-, and low-pressure turboexpander is carried out. The main conclusions of this study are summarized as follows.

- Sobol method describes the important non-dimensional design variables, and their optimal ranges are predicted by developing artificial intelligence models.

- The numerical analysis reveals that the distribution of pressure, Mach number, and temperature contours are uniform with less amount of local variation.

- The splitter blades reduce the intensity of passage vortices, flow separation, and scraping flow phenomenon which increases the isentropic efficiency and power output of the turboexpander.
- The numerical simulation is validated with experimental and numerical results which shows that the present study is reliable.

- The study is helpful in the design and performance prediction of a cryogenic radial turbine and to develop the experimental prototype of a helium turboexpander system.

Acknowledgements The authors sincerely thank Board of Research in Nuclear Sciences (BRNS), Ministry of Human Resource Development (MHRD), Government of India, and National Institute of Technology, Rourkela, for providing the financial support.

\section{Compliance with ethical standards}

Conflict of interest The authors declared that there is no conflict of interest to any person or organization.

\section{References}

1. Li X, Li J, Pan W, Zou Y, Li Q (2019) Investigation on tip leakage flow characteristics of radial inflow micro turbine used in helium liquefying system. Int J Refrig 98:51-60

2. Cho S-Y, Cho C-H, Ahn K-Y, Lee YD (2014) A study of the optimal operating conditions in the organic Rankine cycle using a turbo-expander for fluctuations of the available thermal energy. Energy 64:900-911

3. Wei D, Lu X, Lu Z, Gu J (2007) Performance analysis and optimization of organic Rankine cycle (ORC) for waste heat recovery. Energy Convers Manag 48(4):1113-1119

4. Kumar M, Panda D, Behera SK, Sahoo RK (2019) Experimental investigation and performance prediction of a cryogenic turboexpander using artificial intelligence techniques. Appl Therm Eng 162:114273

5. Ghosh SK, Sahoo RK, Sarangi SK (2010) Experimental performance study of cryogenic turboexpander by using aerodynamic thrust bearing. Appl Therm Eng 30(11-12):1304-1311

6. Ghosh SK, Sahoo R, Sarangi SK (2011) Mathematical analysis for off-design performance of cryogenic turboexpander. J Fluids Eng 133(3):031001

7. Hongli Z, Yu H, Liang C (2009) Experimental study on a small Brayton air refrigerator under $-120^{\circ} \mathrm{C}$. Appl Therm Eng 29(8-9):1702-1706

8. Sam AA, Mondal J, Ghosh P (2017) Effect of rotation on the flow behaviour in a high-speed cryogenic microturbine used in helium applications. Int J Refrig 81:111-122

9. Zhang Z, Li M, Ma Y, Gong X (2015) Experimental investigation on a turbo expander substituted for throttle valve in the subcritical refrigeration system. Energy 79:195-202

10. Davis H, Land ML (1970) Integral turbo compressor-expander system for refrigeration. US patent 3,494,145

11. Song J, Gu C-W, Ren X (2016) Influence of the radial-inflow turbine efficiency prediction on the design and analysis of the organic Rankine cycle (ORC) system. Energy Convers Manag 123:308-316

12. Da Lio L, Manente G, Lazzaretto A (2017) A mean-line model to predict the design efficiency of radial inflow turbines in organic Rankine cycle (ORC) systems. Appl Energy 205:187-209 
13. Han Z, Fan W, Zhao R (2017) Improved thermodynamic design of organic radial-inflow turbine and ORC system thermal performance analysis. Energy Convers Manag 150:259-268

14. Zhai L, Xu G, Wen J, Quan Y, Fu J, Wu H, Li T (2017) An improved modeling for low-grade organic Rankine cycle coupled with optimization design of radial-inflow turbine. Energy Convers Manag 153:60-70

15. Ghosh SK (2008) Experimental and computational studies on cryogenic turboexpander. Ph.D. thesis

16. Suhrmann JF, Peitsch D, Gugau M, Heuer T, Tomm U (2010) Validation and development of loss models for small size radial turbines. In: ASME turbo expo 2010: power for land, sea, and air. American Society of Mechanical Engineers, pp 1937-1949

17. Mounier V, Mendoza LC, Schiffmann J (2017) Thermo-economic optimization of an ORC driven heat pump based on small scale turbomachinery and comparison with absorption heat pumps. Int J Refrig 81:96-110

18. Yu Y, Chen L, Sun F, Wu C (2007) Neural-network based analysis and prediction of a compressor's characteristic performance map. Appl Energy 84(1):48-55

19. Fast M, Assadi M, De S (2009) Development and multi-utility of an ANN model for an industrial gas turbine. Appl Energy 86(1):9-17

20. Bartolini C, Caresana F, Comodi G, Pelagalli L, Renzi M, Vagni $S$ (2011) Application of artificial neural networks to micro gas turbines. Energy Convers Manag 52(1):781-788

21. Tjokroamlnata W, Tan C, Hawthorne W (1994) A design study of radial inflow turbines with splitter blades in three-dimensional flow. In: ASME 1994 international gas turbine and aeroengine congress and exposition. American Society of Mechanical Engineers, pp V001T01A009-V001T01A009

22. Xia J, Wang J, Wang H, Dai Y (2018) Three-dimensional performance analysis of a radial-inflow turbine for an organic Rankine cycle driven by low grade heat source. Energy Convers Manag 169:22-33

23. Aungier RH (2006) Aerodynamic design and performance analysis of exhaust diffusers. ASME Press, New York City

24. Li Y, Ren X-D (2016) Investigation of the organic Rankine cycle (ORC) system and the radial-inflow turbine design. Appl Therm Eng 96:547-554
25. Glassman AJ (1994) Turbine design and application volumes 1, 2, and 3. National Aeronautics and Space Administration (NASA), Washington

26. Barad SG, Ramaiah PV, Giridhar RK, Krishnaiah G (2012) Neural network approach for a combined performance and mechanical health monitoring of a gas turbine engine. Mech Syst Signal Process 27:729-742

27. Jang J-S, Sun C-T (1995) Neuro-fuzzy modeling and control. Proc IEEE 83(3):378-406

28. Schlechtingen M, Santos IF, Achiche S (2013) Using data-mining approaches for wind turbine power curve monitoring: a comparative study. IEEE Trans Sustain Energy 4(3):671-679

29. Ariff M, Salim SM, Cheah SC (2009) Wall y+ approach for dealing with turbulent flow over a surface mounted cube: part 1-low Reynolds number. In: Seventh international conference on CFD in the minerals and process industries, pp 1-6

30. Ansys $C$ (2015) Release 15.0-user manual. ANSYS, Canonsburg

31. Dong B, Xu G, Li T, Quan Y, Zhai L, Wen J (2018) Numerical prediction of velocity coefficient for a radial-inflow turbine stator using R123 as working fluid. Appl Therm Eng 130:1256-1265

32. Lei $Q$, Zhengping $Z$, Huoxing L, Wei $L$ (2010) Upstream wake-secondary flow interactions in the endwall region of high-loaded turbines. Comput Fluids 39(9):1575-1584

33. Kumar M, Sahoo R, Behera S (2018) Design and numerical investigation to visualize the fluid flow and thermal characteristics of non-axisymmetric convergent nozzle. Eng Sci Technol Int J 22:294-312

34. Chakravarty A, Menon RS, Goyal M, Ahmed N (2011) Recent developments in high speed cryogenic turboexpanders at BARC, Mumbai. In: Asian conference on applied superconductivity and cryogenics (New Delhi)

35. Sun W, Chen S, Niu L, Hou Y (2015) Wetness loss prediction for a wet-type cryogenic turboexpander based on 3-D numerical simulation. Appl Therm Eng 91:1032-1039

36. Zangeneh M, Goto A, Harada $\mathrm{H}$ (1998) On the design criteria for suppression of secondary flows in centrifugal and mixed flow impellers. J Turbomach 120(4):723-735

Publisher's Note Springer Nature remains neutral with regard to jurisdictional claims in published maps and institutional affiliations. 